\title{
Development and Evaluation of a Synthetic Opioid Targeted Gas Chromatography Mass Spectrometry (GC-MS) Method
}

\author{
Edward Sisco $^{\mathrm{a}}$, Amber Burns ${ }^{\mathrm{b}}$, Arun S. Moorthy ${ }^{\mathrm{a}}$ \\ aNational Institute of Standards and Technology \\ ${ }^{b}$ Maryland State Police Forensic Sciences Division \\ *edward.sisco@nist.gov, 301-975-2093
}

\begin{abstract}
A method for the targeted confirmation of synthetic opioids and related compounds observed in forensic seized drug analysis was developed and evaluated. An 11-component test solution was used to develop a method that focused on minimizing overlapping retention time acceptance windows and understanding the influence of instrument parameters on reproducibility and sensitivity. Investigated settings included column type, flow rate, temperature program, inlet temperature, source temperature, and tune type. Using a DB-200 column, a 35-minute temperature ramped method was created. It was evaluated against a suite of 222 synthetic opioids and related compounds, and successfully differentiated all but four compound pairs based on retention time or mass spectra. Compared to a general confirmatory method, the targeted method was up to 25 times more sensitive and provided at least a two-fold increase in retention time differences. Analysis of case extracts successfully demonstrated utility of the method and showed no instance of carryover, although the high polarity column required wider retention time windows than other columns. Development of the targeted method is part of a larger effort to better understand the challenges and benefits of different analytical workflows in seized drug analysis.
\end{abstract}

\section{Highlights}

- A method for targeted confirmation of synthetic opioids and related compounds was developed and evaluated.

- The resulting method utilizes a DB-200 column and a 35-minute runtime.

- A total of 222 compounds have been analyzed and four pairs were not differentiable.

- The targeted method was more sensitive and provided better separation than a general confirmatory method.

Keywords: Opioids; GC-MS; Seized Drug; NPS; Method Development 


\section{Introduction}

Opioids are one of the most frequently encountered compound classes in seized drug analysis [1] and present a number of analytical challenges. The rise of synthetic opioids, like fentanyl and fentanyl analogs, over the last decade[2,3] has brought with it a wide variety of compounds that must be identified, confirmed, and differentiated. Many of these compounds are chemically and structurally similar and there are numerous isobaric and isomeric species. A further complication is the high potency of synthetic opioids leading to street samples that are often heavily cut with inert diluents, heroin, and other controlled substances. These low concentrations result in detection challenges. Increased prevalence of the U series opioids[4] and nitazenes[5] represent other subclasses of compounds that can be difficult to confirm, aside from fentanyl.

Generic analytical methods used in seized drug analysis have become less effective for synthetic opioids, necessitating the development of new approaches and methodologies. A large body of research has grown around presumptive analysis of these compounds, either in the laboratory or the field. A number of ambient ionization mass spectrometry (AI-MS) techniques have been demonstrated for opioid analysis, including direct analysis in real time (DART-MS)[6], direct sample analysis (DSA-MS)[7], and paper spray mass spectrometry[8]. Portable mass spectrometers, using a range of ionization techniques, have also been shown[9-11]. Using similar principles, ion mobility spectrometry has been reported to separate and identify some fentanyl compounds[12,13]. Surface enhanced Raman spectroscopy (SERS) has shown potential for opioid analysis[14], as has, for more concentrated samples, Fourier transform infrared (FTIR) [15]. Other novel approaches for presumptive analysis of fentanyl include lateral flow immunoassays (LFIs)[15,16], electrochemistry[17], and electrophoresis[18].

Several techniques for confirmatory analysis of opioids have also been demonstrated including gas chromatography vacuum ultraviolet (GC-VUV)[19,20], GC coupled with infrared detection (GC-IRD)[21], liquid chromatography mass spectrometry (LC-MS)[22], and low-field nuclear magnetic resonance (NMR)[23]. In addition to new instrument-based approaches, several interesting data interpretation techniques and statistical analyses have also been developed. These techniques, which typically utilize mass spectral data, have provided greater insight into fragmentation pathways[24], isobaric fragment ions[25], and profiling capabilities[26-28].

A potential drawback of many of the previously noted tactics is they require the adoption of new technology, which can be challenging in a forensic laboratory setting due to time and financial constraints. A more pragmatic approach to address challenges in the short-term is to develop new methodologies (instrument-based or data-focused) that leverage existing platforms common to the field. Gilbert et al. demonstrated this by highlighting how principal component analysis can be used for the classification of fentanyl analogues based on their gas chromatography mass spectrometry (GC-MS) spectra[29]. Moorthy et al. showed the ability to use GC-MS data combined with hybrid similarity searching[30] and mass spectral similarity mapping to identify previously unseen analogs[31]. 
In this work, we look at complimenting these data-focused approaches by establishing a GC-MS instrumental method specifically for the confirmation of synthetic opioids. The method was developed and evaluated using a previously deployed framework[32] with the goals of (1) minimizing overlapping retention time acceptance windows, (2) understanding how to alter sensitivity of the method without effecting reproducibility, and (3) measuring and documenting the limitations of the method in regards to compound discrimination. Using a test solution consisting of eleven compounds, the method was developed by investigating column type, flow rate, temperature program, inlet, and mass spectrometer source conditions. Once established, the method was evaluated by analyzing over 200 additional opioids and related compounds in addition to a suite of case samples to capture limitations of this approach. Comparisons to an existing general-purpose GC-MS method were also made. Implementation of a targeted method is envisioned to occur alongside information-rich screening tools (such as DART-MS[33], DSA-MS, or Raman spectroscopy[34]) or in instances where there are known compound confirmation difficulties using general-purpose methods.

\section{Materials and Methods}

Approach for Developing Targeted Methods

A previously published framework for the development and evaluation of the targeted synthetic opioid method was used for this work[32]. Briefly, method development began with investigation of the six different column stationary phases to establish which phase provided the best balance of retention time differentiation, sensitivity, and total analysis time (Step 1). Once a stationary phase was chosen, additional studies were completed to evaluate different oven temperature programs and flow rates to attempt to shorten analytical runtimes while maintaining sufficient retention time differences (Step 2). A design of experiments was then used to investigate inlet temperature, split ratio, injection volume, and MS source temperature, and tune types (stune vs atune) to understand tradeoffs with sensitivity and reproducibility (Step 3).

The above studies resulted in a preliminary targeted method to use for a series of evaluation studies, the first of which involved expansion of compounds analyzed using the method. Over 200 synthetic opioids and related compounds were analyzed to determine the effectiveness of the method to separate and detect different compounds (Step 4). The targeted method was then compared to a general confirmatory method currently used at the Maryland State Police Forensic Sciences Division (MSP-FSD) to understand the strengths and weaknesses of the targeted method over a currently used method (Step 5). Finally, a series of adjudicated or mock case samples were analyzed to evaluate usability on the type of extracts encountered in a forensic laboratory (Step 6).

\section{Chemicals}

Unless otherwise stated, all analyses used a test solution custom-made by Cayman Chemical (Ann Arbor, MI, USA). The solution contained eleven compounds: m-fluoroisobutyryl fentanyl (m-FIBF), p-fluoroisobutyryl fentanyl, (pFIBF), fentanyl, cyclopropyl fentanyl, methoxyacetyl fentanyl, crotonyl fentanyl, carfentanil, furanyl fentanyl, etizolam, benzodioxole fentanyl, and noscapine. Each compound was present at a nominal concentration of $100 \mu \mathrm{g}$ $\mathrm{mL}^{-1}$, in methanol. The solution was received as individual $1 \mathrm{~mL}$ ampoules, and a fresh ampoule was used each day studies were run. In addition to the test solution, individual compounds in methanol (at a nominal concentration of 
$100 \mu \mathrm{g} / \mathrm{mL}$ ) were also analyzed using standards obtained from Cayman Chemical. A Fentanyl Analog Screening Kit (FAS Kit) from Cayman Chemical was also used to investigate a broad range of synthetic opioids once the targeted method was developed.

Instrument, Consumables, and Data Analysis

All analyses were completed using an Agilent 7890/5977B GC-MS (Agilent Technologies, Santa Clara, CA, USA). Method parameters varied throughout the project and are discussed throughout the text. Six columns were investigated, all of which had dimensions of $30 \mathrm{~m} \times 0.25 \mathrm{~mm} \times 0.25 \mu \mathrm{m}$, with stationary phases of DB-1UI, DB-5, DB-5UI, DB35, DB-200, and VF-1701ms (Agilent Technologies). Unless otherwise noted, the standard spectral tune (stune) was used along with an MS scan range of $\mathrm{m} / \mathrm{z} 50$ to $\mathrm{m} / \mathrm{z} 450$, a scan speed of $\mathrm{N}=2$, and a threshold of 150 counts.

Data analysis protocols from [32] were employed. MassHunter (Agilent Technologies) was used for chromatographic peak integration and AMDIS (NIST, Gaithersburg, MD, USA) for chromatogram deconvolution and mass spectral quality analysis. Using data from the Scientific Working Group for Seized Drug Analysis (SWGDRUG) MS Library (version 3.6), a custom library consisting of mass spectra of all compounds in the test solution was created for assessing the quality of experimentally collected mass spectra. The full SWGDRUG MS Library was used for Step 6. Additional data analysis parameters can be found elsewhere[32] and in the Supplemental Information. The min-max mass spectral similarity test was used to establish instances where mass spectra of closely eluting compounds could be differentiated. Details of the min-max test are provided elsewhere[32] and in the Supplemental Information.

\section{Results and Discussion}

Column Comparison (Step 1)

Comparison of the six stationary phases was completed by analyzing the test solution, in triplicate, on all columns using the same instrumental parameters (Supplemental Table 1). Peak area, peak height, retention time, and peak width (FWHM) for each compound were extracted along with peak purity measurements (from AMDIS) and mass spectral quality matches (from AMDIS). The 11 compounds in the test solution were also analyzed individually on each column to aid in identifying occurrences of overlapping peaks and changes in elution order. It should also be noted that column dependent variation in elution order was observed and is highlighted in Supplemental Figure 1.

A summary of the results obtained from the different columns are shown in Figure 1. Significant differences in peak areas (Figure 1B) were observed across the six columns, with VF-1701ms providing the lowest integrated peak areas, on average, and DB-35 the greatest. Peak purity, defined as the percentage of the total ion signal within a chromatographic peak that can be attributed to the analyte, also showed differences across the column types (Figure 1C). Purity tended to decrease with later eluting compounds, which was expected due to increased column bleed at higher oven temperatures. The DB-200 and VF-1701ms columns were found to have the most consistent peak purities (lowest standard deviations) across the components of the test mixture, followed by the DB-35 column. Representative chromatograms of the test solution analyzed on all columns are shown in Supplemental Figure 1. 
The weighted mass spectral comparison scores, from AMDIS, for all compounds were also extracted and, in nearly all instances, were equal to or exceeded 85 a.u., out of a possible 100 a.u. Mass spectra obtained using the VF-1701ms column had the lowest average similarity scores and highest standard deviation, at 86.7 a.u. ( \pm 25.1 a.u.), while the spectra from the DB-1 and DB-35 columns performed the best (Supplemental Table 2). Peak widths (Supplemental Table 2) were consistent across all columns, though slightly wider for the VF-1701ms column.

To compare chromatographic separability, the percent differences in retention times between neighboring peaks (\%RTD) were measured, as defined in Equation 1, and are plotted in Figure 1A. The VF-1701ms column was superior as it was the only one that achieved greater than $1 \%$ retention time difference (\%RTD) between all sets of neighboring peaks that eluted - though noscapine and benzodioxole fentanyl did not elute during the 30 min runtime. Elution of all compounds was achieved on the remaining five columns, and all had at least one instance of co-eluting, or nearly co-eluting peaks. On the DB-1UI column, co-elution was observed with furanyl fentanyl and etizolam, and there were four additional pairs of nearly co-eluting peaks. The same five pairs of compounds were problematic on the DB-5 and DB-5UI columns.

$$
\% R T D=\frac{\mid(\text { Retention Time Compound } 1)-(\text { Retention Time Compound } 2) \mid}{(\text { Retention Time Compound } 1)} * 100 \text { (Eqn. 1) }
$$

The DB-1UI, DB-5UI, and DB-5 columns were not explored further due to poor chromatographic separability. The VF-1701ms column was not explored further due to the inability to elute all compounds in the test solution and the high variability in mass spectral library scores. The DB-35 and DB-200 columns both had acceptable mass spectral quality and only two pairs of compounds with \%RTDs of less than $1 \%$. Both columns produced mass spectra with acceptable quality (a similarity score of greater than 90 a.u. when compared to the SWGDRUG library). While both columns were deemed suitable for the development of a targeted method, the DB-200 column was chosen as it has been previously shown to be optimal for the analysis of synthetic cannabinoids[32] and therefore the same column could be employed for both methods. 


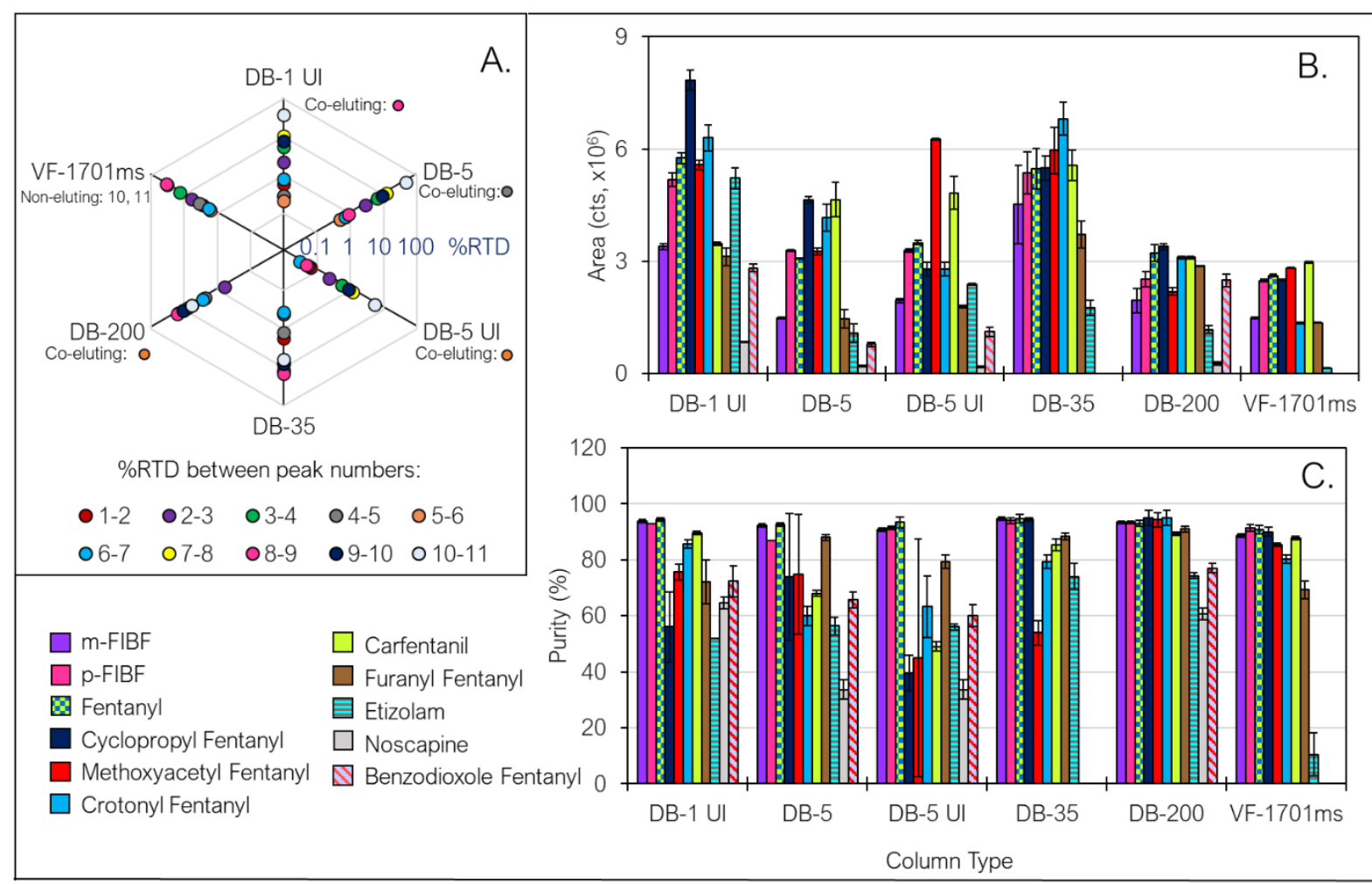

Figure 1. Results of the column comparison study (Step 1). The radar plot (A.) shows the percent retention time difference (\%RTD) for neighboring peaks in the test mixture (sequentially numbered 1 through 11 because of differences in elution order). Points further out on the web indicate better separation. Note the plot is log scale. Average peak areas (B.) and peak purities (C.) for each compound analyzed on each column are also shown. Uncertainties represent the standard deviation of triplicate measurements. Compounds are listed in the elution order when using a DB-5 column. For the DB-35 column, detection of noscapine and benzodioxole fentanyl was only possible using extracted ion chromatograms and therefore they are not included in (B.) or (C.)

Maximizing Retention Time Differences Through Temperature and Flow Parameters (Step 2)

Once a column was chosen, different carrier gas flow rates and oven temperature programs were assessed to identify conditions that allowed for maximized differences in retention times for of test solution compounds while maintaining a reasonable runtime. Isothermal and ramped (single ramps and multiple ramps) temperature programs were evaluated, along with flow rates ranging from $0.8 \mathrm{~mL} \mathrm{~min}^{-1}$ to $2.0 \mathrm{~mL} \mathrm{~min}^{-1}$, as summarized in Table 1 . The isothermal temperature programs at $250^{\circ} \mathrm{C}$ and $290^{\circ} \mathrm{C}$ were unable to sufficiently separate all compounds in the test solution as shown in Table 1 and Supplemental Figure 2. A slow ramp rate $\left(2{ }^{\circ} \mathrm{C} \mathrm{min}^{-1}\right)$ did provide sufficient retention time differences (\%RTD of approximately $1 \%$ or greater) for all components in the test solution, regardless of the flow rate used. Additionally, while the non-isothermal studies initially had a starting temperature of $200{ }^{\circ} \mathrm{C}$, it was found that this could be increased to $230^{\circ} \mathrm{C}$ without affecting compound resolution, allowing for a reduction in runtime. The slow temperature program was found to be necessary to achieve a \%RTD of at least $1 \%$ between the seven earliest eluting compounds. Attempts to reduce the overall runtime by using a multi-step ramp were investigated but did not lead to faster elution of the late eluting compounds. The single $2{ }^{\circ} \mathrm{C} \min ^{-1}$ temperature ramp, starting at $230{ }^{\circ} \mathrm{C}$, was chosen for the targeted method. A mid-range flow rate of $1.2 \mathrm{~mL} \mathrm{~min}^{-1}$ was selected to allow for flexibility in flow rate that could occur when locked retention times were implemented. 
Table 1. Summary of the temperature programs and flow rates studied as well as their respective results. The notation "CEP" denotes instances where there were co-eluting peaks. Uncertainties represent the standard deviation of triplicate measurements.

\begin{tabular}{|c|c|c|c|}
\hline Temperature Program & 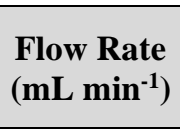 & $\underset{(\%)}{\operatorname{Minimum} \% \mathbf{R T D}}$ & $\begin{array}{c}\text { Maximum } \\
\text { Retention Time } \\
(\text { min })\end{array}$ \\
\hline $250^{\circ} \mathrm{C}$ Isothermal & 2.0 & $0.15( \pm 0.05)$ & 33.14 \\
\hline $290^{\circ} \mathrm{C}$ Isothermal & 0.8 & CEP & 11.50 \\
\hline $290^{\circ} \mathrm{C}$ Isothermal & 2.0 & CEP & 7.75 \\
\hline $\begin{array}{c}200{ }^{\circ} \mathrm{C}-290{ }^{\circ} \mathrm{C} \text { ramping at } \\
2^{\circ} \mathrm{C} \mathrm{min}^{-1}\end{array}$ & 2.0 & $0.97( \pm 0.03)$ & 38.02 \\
\hline $\begin{array}{c}230{ }^{\circ} \mathrm{C}-290{ }^{\circ} \mathrm{C} \text { ramping at } \\
2^{\circ} \mathrm{C} \mathrm{min}^{-1}\end{array}$ & 1.2 & $0.96( \pm 0.07)$ & 24.13 \\
\hline $\begin{array}{c}230{ }^{\circ} \mathrm{C}-290{ }^{\circ} \mathrm{C} \text { ramping at } \\
2^{\circ} \mathrm{C} \mathrm{min}^{-1}\end{array}$ & 2.0 & $1.10( \pm 0.01)$ & 21.45 \\
\hline $\begin{array}{c}230^{\circ} \mathrm{C} \text {, hold } 2 \text { min } \\
\text { Ramp } 1^{\circ} \mathrm{C} \min ^{-1} \text { to } 240{ }^{\circ} \mathrm{C} \\
\text { Hold } 0.5 \text { min } \\
\text { Ramp } 5^{\circ} \mathrm{C} \min ^{-1} \text { to } 290{ }^{\circ} \mathrm{C}\end{array}$ & 1.2 & $1.28( \pm 0.03)$ & 23.91 \\
\hline $\begin{array}{l}230^{\circ} \mathrm{C} \text {, hold } 2 \min \\
\text { Ramp } 1^{\circ} \mathrm{C} \min ^{-1} \text { to } 240{ }^{\circ} \mathrm{C} \\
\text { Hold } 0.5 \min \\
\text { Ramp } 5^{\circ} \mathrm{C} \min ^{-1} \text { to } 290{ }^{\circ} \mathrm{C}\end{array}$ & 2.0 & $1.35( \pm 0.05)$ & 22.23 \\
\hline
\end{tabular}

Assessing Sensitivity and Reproducibility (Step 3)

Once the column type, flow rate, and temperature program were established, a design of experiments (DOE) was completed to determine the effect, if any, of MS source temperature, split ratio, injection volume, and inlet temperature on reproducibility and sensitivity. A two-level $\left(2^{4-1}\right)$ DOE was used, as outlined in Supplemental Table 3, with the experimental levels outlined in Figure 2. Sensitivity was measured using peak area and peak height while reproducibility was measured using the percent relative standard deviation (\%RSD) of peak area, peak height, retention time, and \%RTD across triplicate analyses. The average responses for each measurement were then compared using a Student's T-Test (95\% confidence) to determine which parameters elicited statistically significant differences. The results of the DOE study (Figure 2) showed that MS source temperature and split ratio did not lead to significant differences in sensitivity or reproducibility. A significant difference in peak area $(p=0.046)$ was found for the injection volume, as expected, though the differences were not significant for peak height or for any of the reproducibility measures. Inlet temperature produced statistically different results for reproducibility (\%RSD) measures of peak height $(\mathrm{p}=0.038)$ and retention time $(\mathrm{p}=0.0002)$. In both instances, poorer reproducibility, defined as a higher $\%$ RSD, was obtained using a $200{ }^{\circ} \mathrm{C}$ inlet temperature. A $300{ }^{\circ} \mathrm{C}$ inlet temperature was therefore chosen for the targeted method. A source temperature of $280^{\circ} \mathrm{C}$ was also chosen to minimize potential build-up on the source. Since synthetic opioids are typically present as low weight percentages in street samples, a split ratio of 10:1 was chosen to increase sensitivity. An injection volume of $1 \mu \mathrm{L}$ was chosen to maintain sensitivity without overloading the liner. The split ratio and injection volume, however, could be changed to adjust sensitivity without affecting reproducibility. 

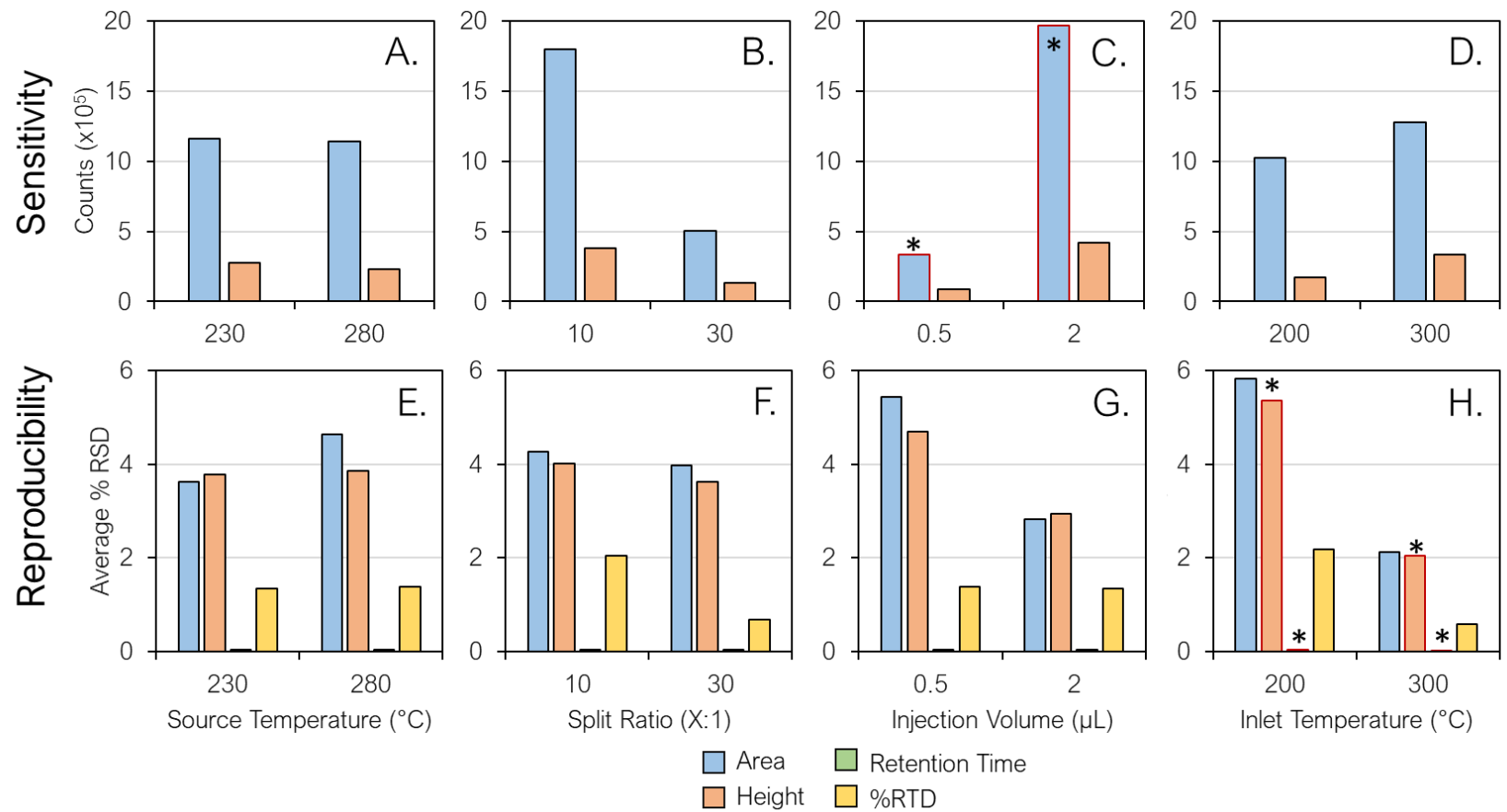

Figure 2. Results of the DOE experiment for source temperature (A. and E.), split ratio (B. and F.), injection volume (C. and G.), and inlet temperature (D. and H.). Peak area (blue) and peak height (orange) are shown in A. through D. while the average \%RSD of peak area (blue), peak height (orange), retention time (green), and \%RTD (yellow) across triplicate injections are shown in E. through $\mathrm{H}$. Boxes with asterisks (*) indicate those where the observed difference was statistically significant at the $95 \%$ confidence level.

The two available tune options, stune and atune, were also examined. Triplicate injections of the test solution were completed using identical methods (consisting of the settings chosen above) except for tune type. Peak areas, shown in Supplemental Figure 3, were nearly identical for all compounds in the test solution for both tune types. The weighted mass spectral scores, obtained by comparing the resulting mass spectra to the SWGDRUG GC-MS library spectra using AMDIS, produced a score of 94 a.u. (out of 100 a.u.) or greater for all components regardless of the tune type used. Since no obvious advantage was shown for either tune type, stune was chosen as it is currently used for casework at the laboratory and is the tune type used for creation of the SWGDRUG library. The final settings chosen for the targeted method are listed in Table 2.

Table 2. Settings for the targeted method. Split ratio and injection volume could be altered, as necessary, to achieve the desired sensitivity. Settings in parentheses indicate settings that were changed after evaluation of the method to address the need for longer runtimes to elute non-volatile compounds (Step 4) and the need to reduce sensitive for the analysis of case samples (Step 6).

\begin{tabular}{|c|c|}
\hline Column & $\begin{array}{c}\text { DB-200 } \\
30 \mathrm{~m} \times 0.25 \mathrm{~mm} \times 0.25 \mu \mathrm{m}\end{array}$ \\
\hline Temperature Program & $\begin{array}{l}\text { 1) } 230{ }^{\circ} \mathrm{C} \text { for } 0.0 \min \\
\text { 2) Ramp at } 2{ }^{\circ} \mathrm{C} \min ^{-1} \text { to } 290{ }^{\circ} \mathrm{C} \\
\text { 3) Hold } 0.0 \mathrm{~min}(5.0 \mathrm{~min})\end{array}$ \\
\hline Flow Rate & $1.2 \mathrm{~mL} \mathrm{~min}^{-1}$ \\
\hline Injection Volume & $1.0 \mu \mathrm{L}$ \\
\hline Inlet Temperature & $300{ }^{\circ} \mathrm{C}$ \\
\hline Split Ratio & 10:1 (20:1) \\
\hline Transfer Line & $300^{\circ} \mathrm{C}$ \\
\hline
\end{tabular}




\begin{tabular}{|c|c|}
\hline Quad Temperature & $150{ }^{\circ} \mathrm{C}$ \\
\hline Source Temperature & $280^{\circ} \mathrm{C}$ \\
\hline Tune Mode & Stune \\
\hline Solvent Delay & $1.3 \mathrm{~min}$ \\
\hline Mass Scan Range & $\mathrm{m} / \mathrm{z} 40-\mathrm{m} / \mathrm{z} 550$ \\
\hline Threshold & 150 \\
\hline Scan Speed & $\mathrm{N}=2$ \\
\hline Total Runtime & $30.0 \mathrm{~min}(35.0 \mathrm{~min})$ \\
\hline
\end{tabular}

\section{Evaluation of the Method with Additional Compounds (Step 4)}

To evaluate and characterize the utility and limitations of the targeted method, an extended panel (Supplemental Table 4) consisting of a wide range of synthetic opioids and commonly co-observed compounds were analyzed. First, each compound was run as single component solution to establish an approximate retention time and to determine if any method modifications were necessary due to elution difficulties. Three fentanyl analogs did not elute in the $30 \mathrm{~min}$ run, resulting in the need to extend the method by an additional $5 \mathrm{~min}$.

After extending the method, it was retention time locked using fentanyl (8.847 min) as the lock compound. The single component solutions were combined into forty mixtures, each containing compounds that had significantly different retention times. Each mixture was measured three times to establish locked retention times and to obtain replicate mass spectra in order to apply the min-max test to closely eluting peaks, as described below. Since some laboratories utilize retention indices, an alkane ladder was also incorporated into the sequence so that retention indices could be calculated. Retention times and retention indices for a subset of the commonly encountered compounds is presented in Table 3, while the full list is presented in Supplemental Table 4.

In addition to establishing retention times and indices, the full dataset was also used to identify which compounds could not be differentiated by retention time or mass spectra. While laboratories use different retention time windows to establish when two compounds are sufficiently separated, a conservative \%RTD window of $2 \%$ was used to identify the largest number of potentially indistinguishable pairs. A total of 599 pairs of compounds (out of 24531 unique pairs of compounds) had retention times within $2 \%$ of one another. The ability to differentiate this subset of compound pairs based on their mass spectra was then examined using the min-max test. While described in detail elsewhere[32], the min-max test compares the similarity scores obtained from replicate mass spectra of two unique compounds to themselves to the similarity scores obtained from mass spectra of the two unique compounds to each other. The result of this approach is a min-max index which represents the difference between the minimum similarity score when comparing replicate spectra of Compound 1 or Compound 2 and the maximum similarity score between spectra from Compound 1 and Compound 2. Possible min-max indices range from -999 a.u. to 999 a.u, where a non-positive value indicates that the two compounds are not differentiable based on their mass spectra.

Of the 599 pairs of compounds that had \%RTDs less than or equal to $2 \%$, only four pairs had non-positive min-max indices (Table 4), indicating that their spectra are not differentiable. Three of the compound pairs (m-methyl Cyclopropyl fentanyl | o-methyl Cyclopropyl Fentanyl, p-fluoro Furanyl Fentanyl 3-furancarboxamide | p-fluoro 
Furanyl Fentanyl, and m-Methylfentanyl | o-Methylfentanyl) were found to be positional isomers and the fourth pair was the acid and free-base forms of Remifentanil. While a non-positive min-max index definitively indicates that the two compounds do not have distinguishable mass spectra, pairs of compounds with index values less than 100 may also produce mass spectra that would be too difficult to differentiate visually. Of the 599 compound pairs that had $\%$ RTDs less than or equal to $2 \%$, an additional 22 pairs, also listed in Table 4, had positive indices less than 100. In nearly all instances, the pairs were positional isomers of compounds that are not frequently encountered. All but one pair (etodesnitazene | isodesnitazene) were fentanyl analogs. While a \%RTD of $2 \%$ was used here to provide a conservative evaluation of the method, laboratories that have tighter windows would have fewer compound pairs that are not differentiable.

Table 3. Retention time (RT), percent retention time difference (\%RTD, as defined by Equation 1), retention time difference (RTD, defined as the difference between a compound and the one listed below), and retention index (RI) for a subset of the frequently seen compounds analyzed by the targeted method. Retention times and indices are the average of three replicates of an approximately $100 \mu \mathrm{g} \mathrm{mL}^{-1}$ solution. Uncertainties represent the standard deviation of three replicates.

\begin{tabular}{|c|c|c|c|c|}
\hline Compound & RT (min) & \%RTD & RTD (min) & RI (a.u.) \\
\hline Tramadol & $2.085( \pm 0.006)$ & 12.3 & 0.257 & 2265 \\
\hline Xylazine & $2.342( \pm 0.011)$ & 2.5 & 0.058 & 2363 \\
\hline o-Desmethyl-cis-Tramadol & $2.400( \pm 0.010)$ & 12.4 & 0.298 & 2386 \\
\hline Norfentanyl & $2.698( \pm 0.005)$ & 0.4 & 0.011 & 2473 \\
\hline Acetyl norfentanyl & $2.709( \pm 0.003)$ & 70.6 & 1.912 & 2483 \\
\hline 4-ANPP & $4.621( \pm 0.004)$ & 17.3 & 0.799 & 2834 \\
\hline AP-238 & $5.420( \pm 0.006)$ & 8.5 & 0.462 & 2929 \\
\hline 2-Methyl AP-237 & $5.882( \pm 0.012)$ & 11.5 & 0.677 & 2980 \\
\hline 6-Monoacetylmorphine & $6.559( \pm 0.007)$ & 3.8 & 0.247 & 3047 \\
\hline U-47700 & $6.806( \pm 0.004)$ & 3.0 & 0.205 & 3071 \\
\hline U-48800 & $7.011( \pm 0.000)$ & 1.4 & 0.099 & 3091 \\
\hline Benzyl Fentanyl & $7.110( \pm 0.002)$ & 11.9 & 0.845 & 3103 \\
\hline Remifentanil & $7.955( \pm 0.000)$ & 3.2 & 0.251 & 3174 \\
\hline m-Fluoroisobutyryl Fentanyl & $8.206( \pm 0.003)$ & 0.4 & 0.035 & 3192 \\
\hline U-49900 & $8.241( \pm 0.003)$ & 0.4 & 0.033 & 3198 \\
\hline Oxycodone & $8.274( \pm 0.008)$ & 3.7 & 0.306 & 3198 \\
\hline FIBF & $8.580( \pm 0.004)$ & 1.1 & 0.098 & 3222 \\
\hline trans-3-methyl Fentanyl & $8.678( \pm 0.002)$ & 1.9 & 0.169 & 3231 \\
\hline Fentanyl & $8.847( \pm 0.009)$ & 1.1 & 0.094 & 3243 \\
\hline Acetyl fentanyl & $8.941( \pm 0.006)$ & 0.1 & 0.005 & 3249 \\
\hline Acrylfentanyl & $8.946( \pm 0.003)$ & 1.7 & 0.151 & 3250 \\
\hline cis-3-methyl Fentanyl & $9.097( \pm 0.002)$ & 1.3 & 0.117 & 3266 \\
\hline Heroin & $9.214( \pm 0.014)$ & 0.1 & 0.012 & 3269 \\
\hline p-Fluorofentanyl & $9.226( \pm 0.003)$ & 1.3 & 0.117 & 3274 \\
\hline Metodesnitazene & $9.343( \pm 0.008)$ & 1.4 & 0.131 & 3279 \\
\hline Butyryl Fentanyl & $9.474( \pm 0.002)$ & 3.1 & 0.293 & 3289 \\
\hline Cyclopropyl Fentanyl & $9.767( \pm 0.002)$ & 2.2 & 0.213 & 3308 \\
\hline Etodesnitazene & $9.980( \pm 0.007)$ & 1.3 & 0.132 & 3333 \\
\hline p-Fluorobutyryl Fentanyl & $10.112( \pm 0.000)$ & 0.1 & 0.014 & 3324 \\
\hline Isodesnitazene & $10.126( \pm 0.007)$ & 0.8 & 0.082 & 3340 \\
\hline Quinine & $10.208( \pm 0.011)$ & 0.1 & 0.008 & 3334 \\
\hline 4'-methyl Acetyl Fentanyl & $10.216( \pm 0.006)$ & 5.1 & 0.519 & 3340 \\
\hline Crotonyl Fentanyl & $10.735( \pm 0.003)$ & 0.5 & 0.049 & 3377 \\
\hline Carfentanil & $10.784( \pm 0.000)$ & 0.1 & 0.016 & 3385 \\
\hline Valeryl Fentanyl & $10.800( \pm 0.002)$ & 10.7 & 1.159 & 3379 \\
\hline Methoxyacetyl Fentanyl & $11.959( \pm 0.004)$ & 8.3 & 0.997 & 3461 \\
\hline Cyclopentyl Fentanyl & $12.956( \pm 0.004)$ & 3.9 & 0.507 & 3320 \\
\hline p-Methoxyfentanyl & $13.463( \pm 0.005)$ & 7.1 & 0.961 & 3551 \\
\hline
\end{tabular}




\begin{tabular}{|c|c|c|c|c|}
\hline $\begin{array}{c}\text { Tetrahydrofuran Fentanyl } \\
\text { Furanyl fentanyl 3-furancarboxamide } \\
\text { isomer }\end{array}$ & $14.424( \pm 0.005)$ & 2.8 & 0.403 & 3607 \\
\hline Furanyl fentanyl & $14.827( \pm 0.004)$ & 2.8 & 0.408 & 3632 \\
\hline Noscapine & $15.235( \pm 0.005)$ & 39.3 & 5.989 & 3652 \\
\hline Flunitazene & $21.224( \pm 0.011)$ & 2.7 & 0.575 & 3977 \\
\hline Etizolam & $21.799( \pm 0.015)$ & 10.5 & 2.298 & 4009 \\
\hline Brorphine & $24.097( \pm 0.010)$ & 3.4 & 0.819 & 4130 \\
\hline Bendioxole Fentanyl & $24.916( \pm 0.023)$ & 8.2 & 2.035 & 4173 \\
\hline Metonitazene & $26.951( \pm 0.002)$ & 0.2 & 0.045 & 4281 \\
\hline Isotonitazene & $26.996( \pm 0.010)$ & 4.0 & 1.079 & 4283 \\
\hline
\end{tabular}

Table 4. Compound pairs with a \%RTD of $2 \%$ or less that also had a min-max index of 100 or less, indicating similarity in their mass spectra. The \%RTD and the min-max index are listed. Compound pairs are listed in order of increasing min-max indices.

\begin{tabular}{|c|c|c|c|}
\hline Compound $1\left(\mathrm{C}_{1}\right)$ & Compound $2\left(\mathrm{C}_{2}\right)$ & $\%$ RTD (\%) & $\begin{array}{l}\text { Min-Max } \\
\text { Index }\end{array}$ \\
\hline m-methyl Cyclopropyl fentanyl & o-methyl Cyclopropyl Fentanyl & 0.66 & -12 \\
\hline Remifentanil & Remifentanil Acid & 1.03 & -2 \\
\hline $\begin{array}{l}\text { p-fluoro Furanyl Fentanyl 3- } \\
\text { furancarboxamide }\end{array}$ & p-fluoro Furanyl Fentanyl & 0.79 & -2 \\
\hline m-Methylfentanyl & o-Methylfentanyl & 0.55 & 0 \\
\hline m-methyl Acetyl fentanyl & o-methyl Acetyl fentanyl & 0.04 & 6 \\
\hline m-methyl Methoxyacetyl Fentanyl & o-methyl Methoxyacetyl Fentanyl & 0.36 & 8 \\
\hline Octfentanil & m-fluoro Methoxyacetyl Fentanyl & 1.03 & 9 \\
\hline o-Fluorofentanyl & m-Fluorofentanyl & 1.81 & 10 \\
\hline o-methyl Furanyl fentanyl & m-methyl Furanyl fentanyl & 0.15 & 13 \\
\hline o-Fluoroisobutyryl Fentanyl & m-Fluoroisobutyryl Fentanyl & 0.65 & 13 \\
\hline $\mathrm{N}-(2 \mathrm{C}-\mathrm{T})$ Fentanyl & N-(2C-T-2) Fentanyl & 0.71 & 26 \\
\hline o-Fluorobutyryl Fentanyl & m-Fluorobutyryl Fentanyl & 1.60 & 26 \\
\hline o-fluoro Furanyl Fentanyl & $\begin{array}{l}\text { p-fluoro Furanyl Fentanyl 3- } \\
\text { furancarboxamide }\end{array}$ & 1.51 & 28 \\
\hline 2',5'-dimethoxy Fentanyl & N-(2C-D) Fentanyl & 1.31 & 33 \\
\hline Etodesnitazene & Isodesnitazene & 1.46 & 39 \\
\hline $\mathrm{N}$-(2,5-DMA) Fentanyl & $\mathrm{N}$-(DOM) Fentanyl & 0.77 & 41 \\
\hline N-(2C-T-4) Fentanyl & N-(2C-T-2) Fentanyl & 1.68 & 49 \\
\hline N-(2C-T-4) Fentanyl & N-(2C-T) Fentanyl & 0.96 & 49 \\
\hline $\mathrm{N}-(2 \mathrm{C}-\mathrm{iP})$ Fentanyl & $\mathrm{N}-(2 \mathrm{C}-\mathrm{E})$ Fentanyl & 0.03 & 54 \\
\hline o-Fluorofentanyl & 2'-fluoro-o-fluorofentanyl & 0.14 & 59 \\
\hline N-(2C-P) Fentanyl & N-(2C-G) Fentanyl & 1.69 & 75 \\
\hline N-(3,4,5-TMA) Fentanyl & N-(DOC) Fentanyl & 0.05 & 75 \\
\hline N-(2C-TFM) Fentanyl & N-(2C-D) Fentanyl & 1.17 & 76 \\
\hline N-(MDA) Fentanyl & N-(6-APB) Fentanyl & 1.73 & 80 \\
\hline 2'-fluoro-o-fluorofentanyl & m-Fluorofentanyl & 1.67 & 88 \\
\hline N-(2-APB) Fentanyl & N-(MDA) Fentanyl & 0.65 & 92 \\
\hline
\end{tabular}

\section{Limits of Detection and Comparison to a General Method (Step 5a)}

Following the expansion of the compound set, the targeted method was compared to a general confirmation method used at MSP-FSD, parameters of which are shown in Supplemental Table 5. Triplicate measurements of the original 11-compound test solution were completed using the targeted method and the general confirmatory method to establish the differences in separation that the targeted method offered. Additionally, the test solution was diluted, volumetrically, to approximate concentrations of $50 \mu \mathrm{g} / \mathrm{mL}, 25 \mu \mathrm{g} / \mathrm{mL}, 10 \mu \mathrm{g} / \mathrm{mL}, 5 \mu \mathrm{g} / \mathrm{mL}$, and $1 \mu \mathrm{g} / \mathrm{mL}$ to measure 
the approximate limit of detection (LOD), defined as the lowest concentration that provided a chromatographic peak with a signal to noise ratio of at least 3:1 and a mass spectral similarity score (from AMDIS) of at least 80 a.u. The results of these experiments are presented in Supplemental Table 6 and representative chromatograms of both methods are shown in Figure 3.

Using the method in Table 2, the targeted method was found to have approximate LODs between 2 and 25 times more sensitive than the general confirmatory method and approximately an order of magnitude higher peak area. When looking at the representative chromatographs in Figure 3 there are multiple instances where the difference in elution time for neighboring compounds was less than $1 \%$ for the general confirmatory method. Methoxyacetyl fentanyl, cyclopropyl fentanyl, and crotonyl fentanyl co-eluted on the general method as did furanyl fentanyl and etizolam. Benzodioxole fentanyl did not elute within the analysis time of the general method. In nearly all instances, the \%RTD between neighboring peaks was larger for the targeted method compared to the general confirmation method.

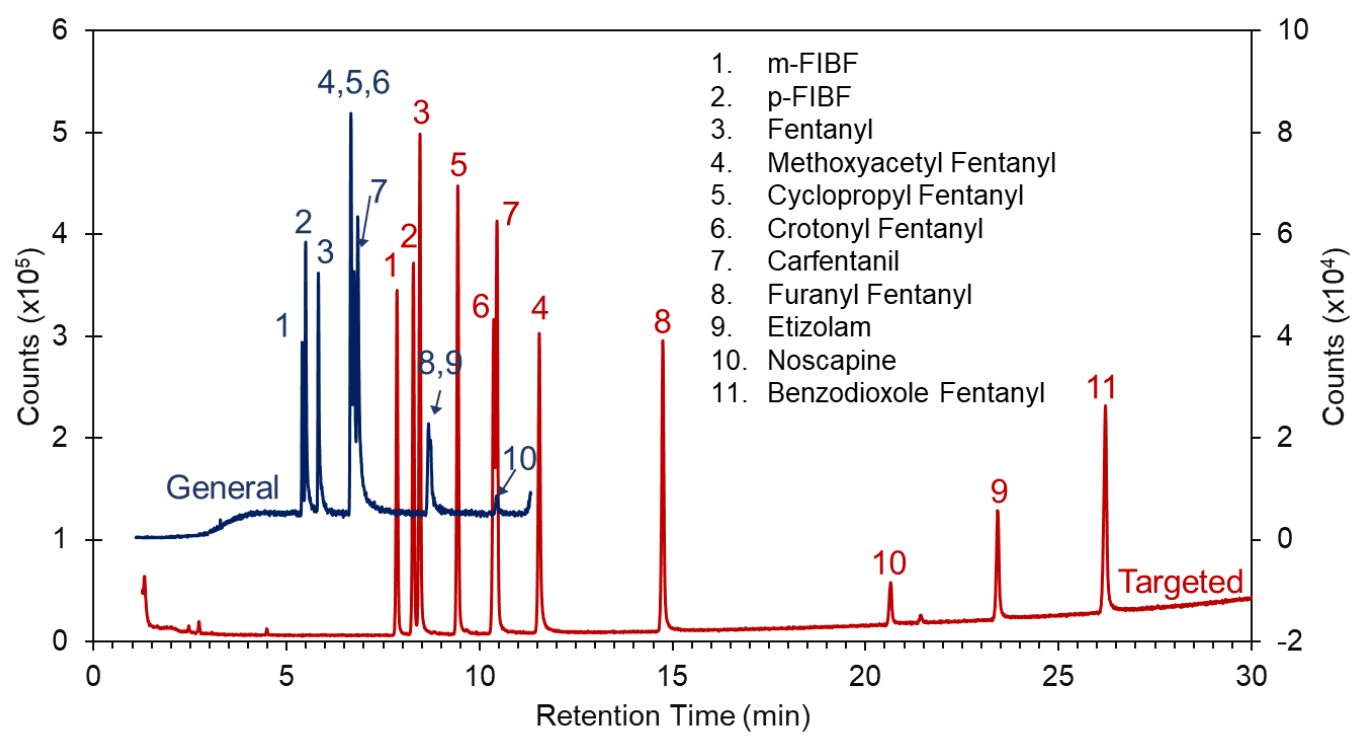

Figure 3. Comparison of the undiluted test solution analyzed using the targeted method (red) and the general confirmatory method (blue). Note the secondary y-axis for the general confirmatory method.

Evaluation of the Method Against Case Samples (Step 6)

The final component of this study looked to evaluate a suite of case extracts using the targeted method to establish whether it was fit-for-purpose or not. Ten previously analyzed case samples were prepared following MSP-FSD protocols whereby approximately $5 \mathrm{mg}$ of powder was dissolved in $1.5 \mathrm{~mL}$ of methanol. Any solids were allowed to settle, and a portion of the extract was transferred into a GC-MS vial for analysis. The identities of the samples are provided in Table 5 and representative chromatograms of the case samples are provided in Supplemental Figure 4.

Extracts were initially run on the targeted method using a 10:1 split ratio which resulted in broad and sometime saturated peaks from the diluents. The split ratio was reduced to 20:1 and was found to provide sufficient reduction in diluent signal without compromising detection of the peaks of interest. In all instances, the synthetic opioids were 
readily detected and returned mass spectral similarity scores - compared to the SWGDRUG library (v3.6) - of 89 a.u. or greater. Retention times were found to be within $2 \%$ of the measured standards in all instances, even given the wide variations in concentrations and the fact that the standards were measured using the lower, 10:1, split ratio. Retention time variation was greater than has been reported[35] using other methods and is likely due to concentration differences coupled with the use of a higher polarity column. Carryover was not observed. In addition to allowing for detection of opioids, the method was able to readily detect many of the cutting agents and other controlled substances present in the extracts. The results of this study highlight the utility of the method for case analysis, and the laboratory is working on fully validating it for casework.

Table 5. Identities and results from the analysis of representative case samples. Samples originated from powders unless otherwise noted. Standard retention times and MS match scores are provided for the synthetic opioids and related compounds only. MS match scores were obtained by searching against the SWGDRUG Library (v3.6).

\begin{tabular}{|c|c|c|c|c|c|}
\hline Case Contents & $\begin{array}{c}\text { Sample } \\
\text { RT (min) }\end{array}$ & $\begin{array}{l}\text { Standard } \\
\text { RT (min) }\end{array}$ & $\begin{array}{c}\% \text { RTD } \\
\text { (relative } \\
\text { to } \\
\text { standard) }\end{array}$ & $\begin{array}{l}\text { MS Match } \\
\text { Score } \\
\text { (Weighted) }\end{array}$ & $\begin{array}{c}\text { Opioid Peak } \\
\text { Height } \\
(\text { Count/s })\end{array}$ \\
\hline N-methyl Norfentanyl & 2.453 & 2.470 & $0.69 \%$ & 98 & $1.5 \times 10^{7}$ \\
\hline Mannitol & 2.196 & & & & $5.8 \times 10^{6}$ \\
\hline Caffeine & 2.427 & & & & $1.0 \times 10^{6}$ \\
\hline Heroin & 9.207 & 9.214 & $0.08 \%$ & 99 & $1.1 \times 10^{5}$ \\
\hline Cyclopropyl Fentanyl & 9.740 & 9.767 & $0.28 \%$ & 97 & $6.4 \times 10^{5}$ \\
\hline Phenyl Fentanyl & 17.811 & 18.022 & $1.17 \%$ & 95 & $1.4 \times 10^{5}$ \\
\hline Mannitol & 2.383 & & & & $3.4 \times 10^{6}$ \\
\hline Fentanyl & 8.755 & 8.847 & $1.04 \%$ & 97 & $2.7 \times 10^{6}$ \\
\hline Acetyl Fentanyl & 8.906 & 8.941 & $0.39 \%$ & 94 & $1.6 \times 10^{6}$ \\
\hline Melatonin & 11.394 & & & & $1.1 \times 10^{6}$ \\
\hline Mannitol & 2.220 & & & & $4.5 \times 10^{6}$ \\
\hline Caffeine & 2.424 & & & & $2.5 \times 10^{7}$ \\
\hline 6-Monoacetylmorphine & 6.556 & 6.559 & $0.05 \%$ & 98 & $3.7 \times 10^{6}$ \\
\hline FIBF & 8.544 & 8.580 & $0.42 \%$ & 97 & $2.9 \times 10^{6}$ \\
\hline Fentanyl & 8.748 & 8.847 & $1.12 \%$ & 97 & $1.0 \times 10^{6}$ \\
\hline Acetyl Fentanyl & 8.899 & 8.941 & $0.47 \%$ & 94 & $4.7 \times 10^{6}$ \\
\hline Heroin & 9.243 & 9.214 & $0.31 \%$ & 98 & $1.1 \times 10^{7}$ \\
\hline Fentanyl & 8.707 & 8.847 & $1.58 \%$ & 97 & $1.5 \times 10^{6}$ \\
\hline XLR11 & 9.476 & & & & $1.2 \times 10^{7}$ \\
\hline XLR Degradant & 10.216 & & & & $7.3 \times 10^{5}$ \\
\hline Dibutylone & 1.893 & & & & $2.5 \times 10^{5}$ \\
\hline Fentanyl & 8.724 & 8.847 & $1.39 \%$ & & $3.4 \times 10^{5}$ \\
\hline JWH-250 & 15.188 & & & 97 & $5.3 \times 10^{6}$ \\
\hline AP-238 & 5.454 & 5.420 & $0.63 \%$ & $97^{*}$ & $1.7 \times 10^{7}$ \\
\hline Phenacetin & 2.082 & & & & $7.9 \times 10^{4}$ \\
\hline Mannitol & 2.150 & & & & $6.2 \times 10^{5}$ \\
\hline Xylazine & 2.350 & & & & $8.7 \times 10^{4}$ \\
\hline Caffeine & 2.412 & & & & $8.4 \times 10^{6}$ \\
\hline Lidocaine & 2.824 & & & & $4.4 \times 10^{4}$ \\
\hline 6-Monoacetylmorphine & 6.527 & 6.559 & $0.49 \%$ & 89 & $3.9 \times 10^{4}$ \\
\hline Fentanyl & 8.689 & 8.847 & $1.78 \%$ & 97 & $4.2 \times 10^{4}$ \\
\hline Mannitol & 2.144 & & & & $4.5 \times 10^{5}$ \\
\hline Caffeine & 2.412 & & & & $9.4 \times 10^{6}$ \\
\hline Cocaine & 3.624 & & & & $3.7 \times 10^{4}$ \\
\hline Acetylcodeine & 6.061 & & & & $1.6 \times 10^{5}$ \\
\hline
\end{tabular}




\begin{tabular}{|c|c|c|c|c|c|}
\hline 6-Monoacetylmorphine & 6.527 & 6.559 & $0.49 \%$ & 98 & $3.9 \times 10^{5}$ \\
FIBF & 8.526 & 8.580 & $0.63 \%$ & 98 & $3.5 \times 10^{5}$ \\
Fentanyl & 8.695 & 8.847 & $1.72 \%$ & 97 & $6.6 \times 10^{5}$ \\
Acetyl Fentanyl & 8.858 & 8.941 & $0.92 \%$ & 94 & $2.4 \times 10^{5}$ \\
Heroin & 9.202 & 9.214 & $0.13 \%$ & 98 & $5.0 \times 10^{6}$ \\
Noscapine & 21.150 & 21.224 & $0.35 \%$ & 96 & $4.0 \times 10^{4}$ \\
\hline N-methyl Cyclopropyl norfentanyl & 2.662 & 2.714 & $1.91 \%$ & 97 & $2.4 \times 10^{6}$ \\
\hline
\end{tabular}

*AP-238 is not in the SWGDRUG Library v3.6. Comparison score was obtained using v3.9.

\section{Conclusion}

This paper presents a GC-MS method (Table 2) that was developed specifically for the confirmatory analysis of synthetic opioids and related compounds using a previously established framework. The method was substantially different than the general confirmatory method employed at MSP-FSD and used a different stationary phase, MS source temperature, oven program, and inlet temperature. It was demonstrated that MS source temperature, split ratio, and injection volume could be altered within the ranges studied without affecting the reproducibility of the method. While the method was substantially longer than the general confirmatory method, it did allow for far greater retention time differences and detection of all compounds in the test solution. The developed method was evaluated using a suite of over 200 compounds and only four pairs of compounds were found to not be differentiable by either retention time or mass spectra. Current efforts are looking at identifying whether or not the implementation of targeted methods, such as this one, coupled with screening by direct analysis in real time mass spectrometry (DART-MS) provides measurable benefits over traditional workflows. Other ongoing efforts are focused on the development of additional targeted methods (stimulants, tryptamines, and benzodiazepines) and further study of the min-max spectral comparison test. Additional data to support this work, as well as updates to panel of compounds analyzed, can be found here[36].

\section{Disclaimer}

Certain commercial products are identified in order to adequately specify the procedure; this does not imply endorsement or recommendation by NIST, nor does it imply that such products are necessarily the best available for the purpose.

Certain commercial products are identified in order to adequately specify the procedure; this does not imply endorsement or recommendation by the Maryland State Police, nor does it imply that such products are necessarily the best available for the purpose.

A portion of this work was supported by Award No. 2018-DU-BX-0165, awarded by the National Institute of Justice, Office of Justice Programs, U.S. Department of Justice. The opinions, findings, and conclusions or recommendations expressed in this publication/program/exhibition are those of the author(s) and do not necessarily reflect those of the Department of Justice.

\section{References}

[1] U.S. Drug Enforcement Administration, Diversion Control Division, National Forensic Laboratory Information System: NFLIS-Drug 2019 Annual Report, U.S. Drug Enforcement Administration, Springfield, VA, 2020.

[2] D. Ciccarone, Fentanyl in the US heroin supply: A rapidly changing risk environment, Int. J. Drug Policy. 46 (2017) 107-111. https://doi.org/10.1016/j.drugpo.2017.06.010.

[3] P. Armenian, K.T. Vo, J. Barr-Walker, K.L. Lynch, Fentanyl, fentanyl analogs and novel synthetic opioids: A comprehensive review, Neuropharmacology. 134 (2018) 121-132. https://doi.org/10.1016/j.neuropharm.2017.10.016. 
[4] M.H. Baumann, G. Tocco, D.M. Papsun, A.L. Mohr, M.F. Fogarty, A.J. Krotulski, U-47700 and Its Analogs: Non-Fentanyl Synthetic Opioids Impacting the Recreational Drug Market, Brain Sci. 10 (2020) 895. https://doi.org/10.3390/brainsci10110895.

[5] I. Ujváry, R. Christie, M. Evans-Brown, A. Gallegos, R. Jorge, J. de Morais, R. Sedefov, DARK Classics in Chemical Neuroscience: Etonitazene and Related Benzimidazoles, ACS Chem. Neurosci. 12 (2021) 1072 1092. https://doi.org/10.1021/acschemneuro.1c00037.

[6] E. Sisco, J. Verkouteren, J. Staymates, J. Lawrence, Rapid detection of fentanyl, fentanyl analogues, and opioids for on-site or laboratory based drug seizure screening using thermal desorption DART-MS and ion mobility spectrometry, Forensic Chem. 4 (2017) 108-115. https://doi.org/10.1016/j.forc.2017.04.001.

[7] A. Moore, J. Foss, M. Juhascik, S. Botch-Jones, F. Kero, Rapid screening of opioids in seized street drugs using ambient ionization high resolution time-of-flight mass spectrometry, Forensic Chem. 13 (2019) 100149. https://doi.org/10.1016/j.forc.2019.100149.

[8] I. W. De Silva, A.N. Couch, G.F. Verbeck, Paper Spray Mass Spectrometry Utilized with a Synthetic Microporous Polyolefin Silica Matrix Substrate in the Rapid Detection and Identification of More than 190 Synthetic Fentanyl Analogs, J. Am. Soc. Mass Spectrom. 32 (2021) 420-428. https://doi.org/10.1021/jasms.0c00250.

[9] T.R. Fiorentin, B.K. Logan, D.M. Martin, T. Browne, E.F. Rieders, Assessment of a portable quadrupole-based gas chromatography mass spectrometry for seized drug analysis, Forensic Sci. Int. 313 (2020) 110342. https://doi.org/10.1016/j.forsciint.2020.110342.

[10] M. Kang, R. Lian, X. Zhang, Y. Li, Y. Zhang, Y. Zhang, W. Zhang, Z. Ouyang, Rapid and on-site detection of multiple fentanyl compounds by dual-ion trap miniature mass spectrometry system, Talanta. 217 (2020) 121057. https://doi.org/10.1016/j.talanta.2020.121057.

[11] J.V. Abonamah, B.A. Eckenrode, M. Moini, On-site detection of fentanyl and its derivatives by field portable nano-liquid chromatography-electron lonization-mass spectrometry (nLC-EI-MS), Forensic Chem. 16 (2019) 100180. https://doi.org/10.1016/j.forc.2019.100180.

[12] H. Zaknoun, M.-J. Binette, M. Tam, Analyzing fentanyl and fentanyl analogues by ion mobility spectrometry, Int. J. Ion Mobil. Spectrom. 22 (2019) 1-10. https://doi.org/10.1007/s12127-019-00244-0.

[13] J. R. Verkouteren, J. Lawrence, R. Michael Verkouteren, E. Sisco, Method for evaluating ion mobility spectrometers for trace detection of fentanyl and fentanyl-related substances, Anal. Methods. 11 (2019) 60436052. https://doi.org/10.1039/C9AY02174D.

[14] A. Haddad, M.A. Comanescu, O. Green, T.A. Kubic, J.R. Lombardi, Detection and Quantitation of Trace Fentanyl in Heroin by Surface-Enhanced Raman Spectroscopy, Anal. Chem. 90 (2018) 12678-12685. https://doi.org/10.1021/acs.analchem.8b02909.

[15] K. McCrae, S. Tobias, C. Grant, M. Lysyshyn, R. Laing, E. Wood, L. Ti, Assessing the limit of detection of Fourier-transform infrared spectroscopy and immunoassay strips for fentanyl in a real-world setting, Drug Alcohol Rev. 39 (2020) 98-102. https://doi.org/10.1111/dar.13004.

[16] Daniel J. Angelini, Tracey D. Biggs, Michele N. Maughan, Michael G. Feasel, Edward Sisco, Jennifer W. Sekowski, Evaluation of a lateral flow immunoassay for the detection of the synthetic opioid fentanyl, Forensic Sci. Int. Submitted (n.d.).

[17] C.E. Ott, H. Cunha-Silva, S.L. Kuberski, J.A. Cox, M.J. Arcos-Martínez, L.E. Arroyo-Mora, Electrochemical detection of fentanyl with screen-printed carbon electrodes using square-wave adsorptive stripping voltammetry for forensic applications, J. Electroanal. Chem. 873 (2020) 114425. https://doi.org/10.1016/j.jelechem.2020.114425.

[18] S.T. Krauss, D. Ross, T.P. Forbes, Separation and Detection of Trace Fentanyl from Complex Mixtures Using Gradient Elution Moving Boundary Electrophoresis, Anal. Chem. 91 (2019) 13014-13021. https://doi.org/10.1021/acs.analchem.9b03083.

[19] S. Buchalter, I. Marginean, J. Yohannan, I.S. Lurie, Gas chromatography with tandem cold electron ionization mass spectrometric detection and vacuum ultraviolet detection for the comprehensive analysis of fentanyl analogues, J. Chromatogr. A. 1596 (2019) 183-193. https://doi.org/10.1016/j.chroma.2019.03.011.

[20] Z.R. Roberson, H.C. Gordon, J.V. Goodpaster, Instrumental and chemometric analysis of opiates via gas chromatography-vacuum ultraviolet spectrophotometry (GC-VUV), Anal. Bioanal. Chem. 412 (2020) 11231128. https://doi.org/10.1007/s00216-019-02337-5.

[21] A.D. Winokur, L.M. Kaufman, J.R. Almirall, Differentiation and identification of fentanyl analogues using GCIRD, Forensic Chem. 20 (2020) 100255. https://doi.org/10.1016/j.forc.2020.100255.

[22] Y. Zhang, Z. Sheng, Z. Hua, C. Liang, Z. Cai, R. Wang, Y. Zhang, Simultaneous separation and determination of 32 fentanyl-related substances, including seven sets of isomeric fentanyl analogues, by ultra-high- 
performance liquid chromatography coupled with high-resolution mass spectrometry, J. Sep. Sci. 43 (2020) 3735-3747. https://doi.org/10.1002/jssc.202000168.

[23] J. Duffy, A. Urbas, M. Niemitz, K. Lippa, I. Marginean, Differentiation of fentanyl analogues by low-field NMR spectroscopy, Anal. Chim. Acta. 1049 (2019) 161-169. https://doi.org/10.1016/j.aca.2018.12.014.

[24] J.T. Davidson, Z.J. Sasiene, G.P. Jackson, The influence of chemical modifications on the fragmentation behavior of fentanyl and fentanyl-related compounds in electrospray ionization tandem mass spectrometry, Drug Test. Anal. 12 (2020) 957-967. https://doi.org/10.1002/dta.2794.

[25] J.T. Davidson, Z.J. Sasiene, G.P. Jackson, The characterization of isobaric product ions of fentanyl using multistage mass spectrometry, high-resolution mass spectrometry and isotopic labeling, Drug Test. Anal. 12 (2020) 496-503. https://doi.org/10.1002/dta.2758.

[26] I.S. Lurie, A.L. Berrier, J.F. Casale, R. Iio, J.S. Bozenko, Profiling of illicit fentanyl using UHPLC-MS/MS, Forensic Sci. Int. 220 (2012) 191-196. https://doi.org/10.1016/j.forsciint.2012.02.024.

[27] B.P. Mayer, A.J. DeHope, D.A. Mew, P.E. Spackman, A.M. Williams, Chemical Attribution of Fentanyl Using Multivariate Statistical Analysis of Orthogonal Mass Spectral Data, Anal. Chem. 88 (2016) 4303-4310. https://doi.org/10.1021/acs.analchem.5b04434.

[28] L. Mörén, J. Qvarnström, M. Engqvist, R. Afshin-Sander, X. Wu, J. Dahlén, C. Löfberg, A. Larsson, A. Östin, Attribution of fentanyl analogue synthesis routes by multivariate data analysis of orthogonal mass spectral data, Talanta. 203 (2019) 122-130. https://doi.org/10.1016/j.talanta.2019.05.025.

[29] N. Gilbert, R.E. Mewis, O.B. Sutcliffe, Classification of fentanyl analogues through principal component analysis (PCA) and hierarchical clustering of GC-MS data, Forensic Chem. 21 (2020) 100287. https://doi.org/10.1016/j.forc.2020.100287.

[30] A.S. Moorthy, W.E. Wallace, A.J. Kearsley, D.V. Tchekhovskoi, S.E. Stein, Combining Fragment-Ion and Neutral-Loss Matching during Mass Spectral Library Searching: A New General Purpose Algorithm Applicable to Illicit Drug Identification, Anal. Chem. 89 (2017) 13261-13268. https://doi.org/10.1021/acs.analchem.7b03320.

[31] A.S. Moorthy, A.J. Kearsley, W.G. Mallard, W.E. Wallace, Mass spectral similarity mapping applied to fentanyl analogs, Forensic Chem. 19 (2020) 100237. https://doi.org/10.1016/j.forc.2020.100237.

[32] E. Sisco, A. Burns, A.S. Moorthy, Development and Evaluation of a Synthetic Cathinone Targeted Gas Chromatography Mass Spectrometry (GC-MS) Method, J. Forensic Sci. Just Accepted. (n.d.). https://doi.org/10.1111/1556-4029.14789.

[33] E. Sisco, T.P. Forbes, Forensic applications of DART-MS: A review of recent literature, Forensic Chem. 22 (2021) 100294. https://doi.org/10.1016/j.forc.2020.100294.

[34] K.C. Doty, C.K. Muro, J. Bueno, L. Halámková, I.K. Lednev, What can Raman spectroscopy do for criminalistics?, J. Raman Spectrosc. 47 (2016) 39-50. https://doi.org/10.1002/jrs.4826.

[35] J.T. Davidson, B.J. Lum, G. Nano, G.P. Jackson, Comparison of measured and recommended acceptance criteria for the analysis of seized drugs using Gas Chromatography-Mass Spectrometry (GC-MS), Forensic Chem. 10 (2018) 15-26. https://doi.org/10.1016/j.forc.2018.07.001.

[36] E. Sisco, Data Supporting the Development of Targeted GC-MS Methods for Seized Drug Analysis, (2021) 3 files, 4.07 MB. https://doi.org/10.18434/MDS2-2367. 


\section{Supplemental Information: \\ Development and Evaluation of a Synthetic Opioid Targeted Gas Chromatography Mass Spectrometry (GC-MS) Method}

\section{Additional Information on Data Analysis}

All datafiles were analyzed using MassHunter (Agilent Technologies) for chromatographic analysis and AMDIS (NIST) for mass spectral analysis. Peak integration in MassHunter was completed using the Agile2 integrator. In AMDIS, a "Simple" analysis of the data files was used along with a component width of 12, a minimum match factor of 40, medium resolution, medium sensitivity, and medium shape requirements. For retention index calculation, an alkane list was loaded into the RI Calibration File. From the AMDIS results, the weighted match factor, peak purity, and, when applicable, the retention indices were obtained.

\section{Additional Information on the Min-Max Test}

An internally developed min-max match factor comparison test was used as an objective and automated way to classify the mass spectra of closely eluting compounds. The comparison test is first described in (1), but, briefly, the difference between the minimum match factor computed between replicate spectra of the same compounds and the maximum match factor computed between all spectra of the two different compounds is computed as the min-max index. The min-max index employs identity match factors, a numerical estimate of similarity between a pair of mass spectra, as described in (2). Confidence that the two compounds are distinguishable via their mass spectra grows as min-max indices increase. Due to its use of extreme values (min and max), the min-max test may behave undesirably if provided with incorrect measurements of one or both target compounds. This is one of our preliminary implementations of the min-max test; additional development and evaluation is on-going. Alternative methods for classifying spectra may also be appropriate for discrimination, including using a match factor threshold between single spectra representing each compound, clustering using all replicates of each compound, or even visual inspection if automation is not required.

\section{References:}

1. Sisco E, Burns A, Moorthy AS. Development of a Targeted Gas Chromatography Mass Spectrometry (GC-MS) Method for Analysis of Synthetic Cannabinoids. Unpublished Work.

2. Moorthy AS, Kearsley AJ. Pattern Similarity Measures Applied to Mass Spectra. In: Cruz M, Pares C, Quintela P, editors. Progress in Industrial Mathematics: Success Stories: The Industry and the Academia Points of View. Springer, 2021;43-53. 

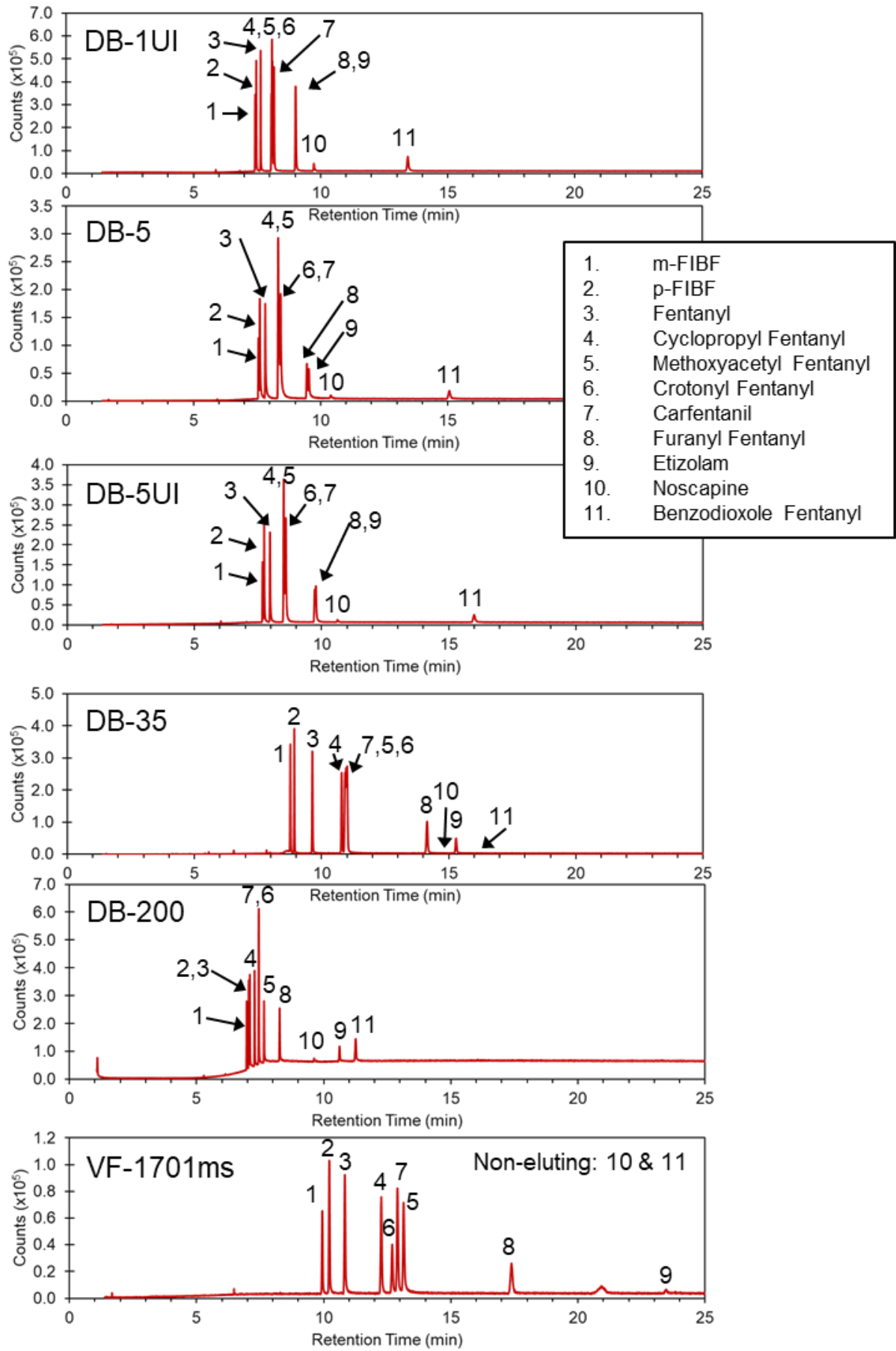

Supplemental Figure 1. Representative chromatograms of the test solution analyzed on each column using the method described in Supplemental Table 1 (Step 1). Note that for the DB-35 column, detection of noscapine and benzdioxole fentanyl was only possible using extracted ion chromatograms. 


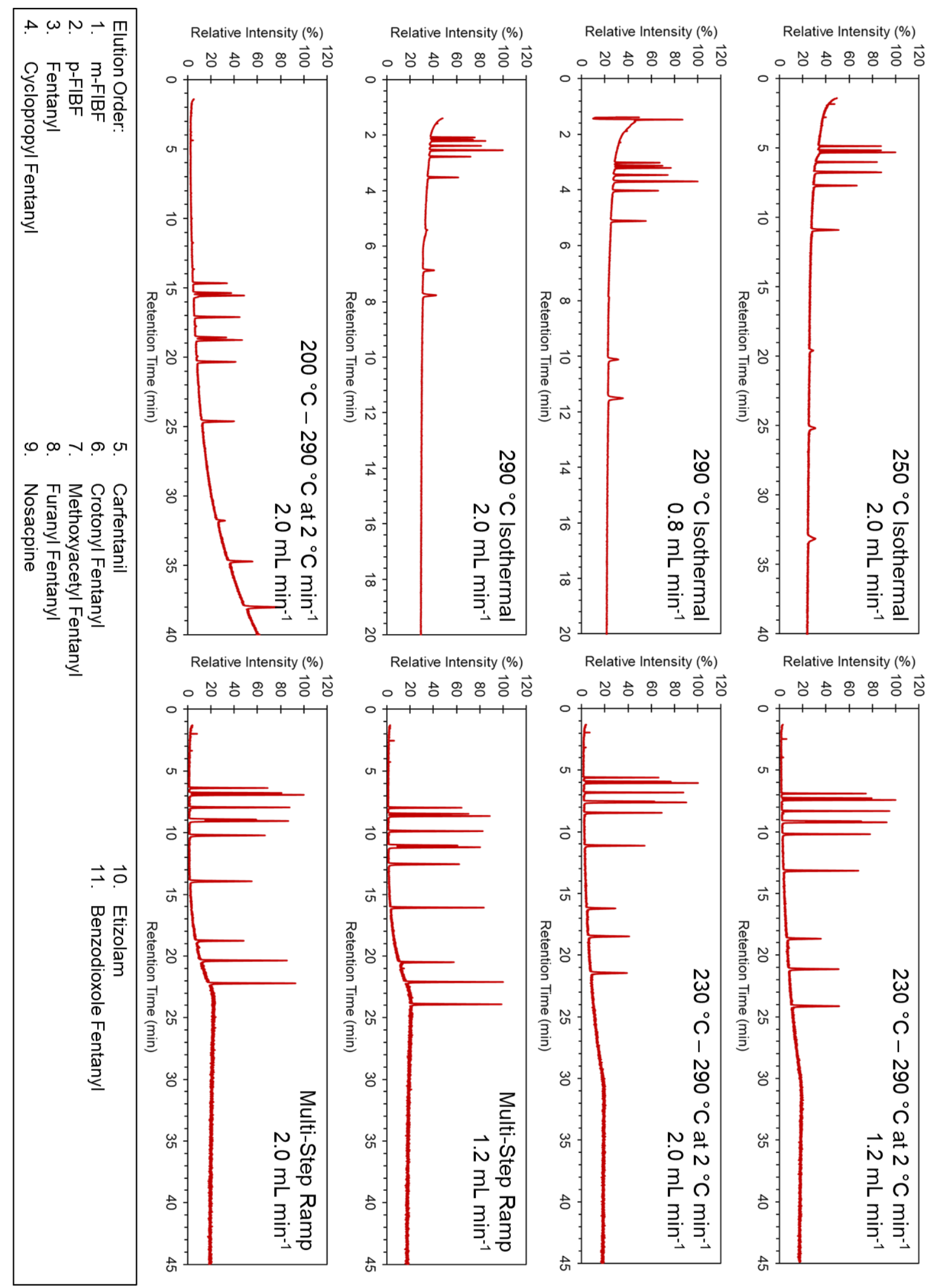

Supplemental Figure 2. Representative chromatograms of the test solution analyzed at each of the temperature and flow program settings investigated in Table 1 (Step 2). 


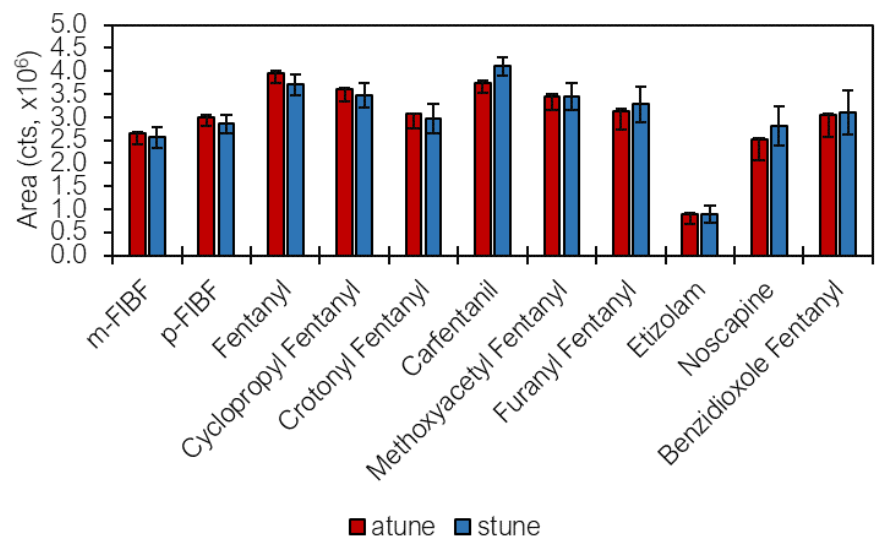

Supplemental Figure 3. Comparison of peak areas obtained when analyzing the test solution using atune (red) and stune (blue). Uncertainties represent the standard deviation of triplicate measurements (Step 3).
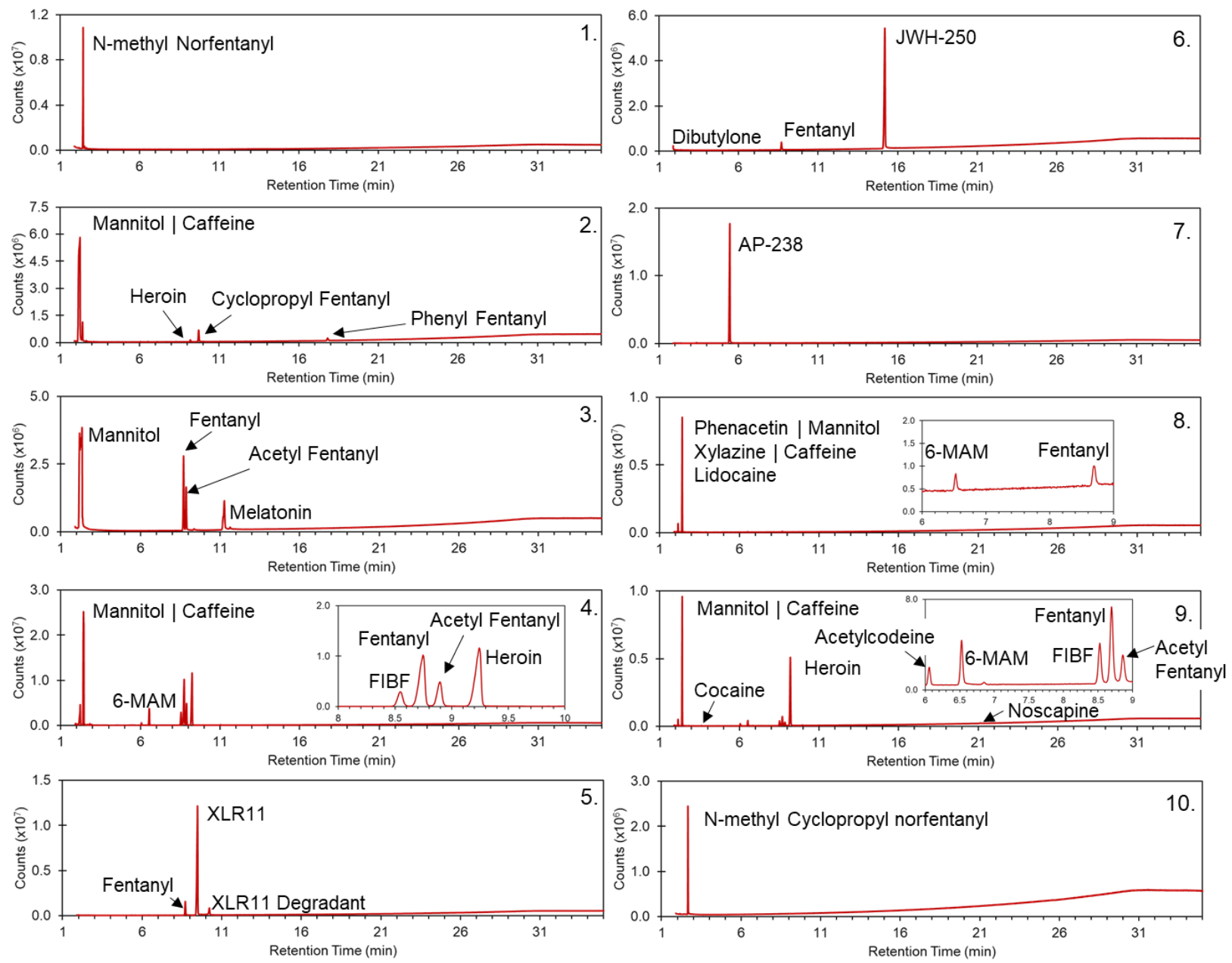

Supplemental Figure 4. Chromatographs of the ten adjudicated or mock case samples (Step 6). 
Supplemental Table 1. Parameters of the method used for the column comparison studies (Step 1).

\begin{tabular}{|l|c|}
\hline Temperature Program & $\begin{array}{c}1) \quad 100{ }^{\circ} \mathrm{C} \text { for } 0 \mathrm{~min} \\
\text { Ramp at } 30{ }^{\circ} \mathrm{C} / \mathrm{min} \text { to } 300{ }^{\circ} \mathrm{C} \\
\text { Hold for } 24 \mathrm{~min}\end{array}$ \\
\hline Flow Rate & $1.8 \mathrm{~mL} / \mathrm{min}(\mathrm{Constant} \mathrm{Flow})$ \\
\hline Injection Volume & $1 \mu \mathrm{L}$ \\
\hline Inlet Temperature & $275^{\circ} \mathrm{C}$ \\
\hline Split Ratio & $30: 1$ \\
\hline Transfer Line & $30{ }^{\circ} \mathrm{C}$ \\
\hline Quad Temperature & $150{ }^{\circ} \mathrm{C}$ \\
\hline Source Temperature & $230{ }^{\circ} \mathrm{C}$ \\
\hline Tune Mode & stune \\
\hline Solvent Delay & $1.30 \mathrm{~min}$ \\
\hline Mass Scan Range & $m / z 40$ to $\mathrm{m} / \mathrm{z} 550$ \\
\hline Threshold & 150 \\
\hline Scan Speed & $\mathrm{N}=2$ \\
\hline Total Run Time & $30.667 \mathrm{~min}$ \\
\hline
\end{tabular}

Supplemental Table 2. Summary results of some key metrics for the different column types examined (Step 1). Uncertainties indicate the standard deviation of averages obtained for each of the detectable compounds in the test solution. The notation "CEP" denotes instances where there were overlapping co-eluting peaks. The notation "NEC" denotes non-eluting compounds.

\begin{tabular}{|c|c|c|c|c|}
\hline Column Type & $\begin{array}{c}\text { Max RT } \\
(\mathbf{m i n})\end{array}$ & $\begin{array}{c}\text { Min \%RTD } \\
(\%)\end{array}$ & $\begin{array}{c}\text { Avg. MS } \\
\text { Match Score }\end{array}$ & $\begin{array}{c}\text { Avg. Peak } \\
\text { Width (min) }\end{array}$ \\
\hline DB-1 UI & 13.42 & CEP & $96.4( \pm 2.0)$ & $0.09( \pm 0.07)$ \\
\hline DB-5 & 15.05 & CEP & $89.5( \pm 10.4)$ & $0.12( \pm 0.07)$ \\
\hline DB-5 UI & 16.00 & CEP & $93.6( \pm 3.4)$ & $0.12( \pm 0.09)$ \\
\hline DB-35 & 16.24 & 0.4 & $96.0( \pm 2.7)$ & $0.14( \pm 0.06)$ \\
\hline DB-200 & 11.29 & CEP & $92.2( \pm 7.4)$ & $0.12( \pm 0.09)$ \\
\hline VF-1701ms & NEC & 1.6 & $86.7( \pm 25.1)$ & $0.21( \pm 0.05)$ \\
\hline
\end{tabular}

Supplemental Table 3. Experimental settings used in the $2^{4-1}$ design of experiment (DOE) study (Step 3).

\begin{tabular}{|c|c|c|c|c|}
\hline Setting \# & $\begin{array}{c}\text { MS Source } \\
\text { Temperature }\left({ }^{\circ} \mathbf{C}\right)\end{array}$ & Split Ratio $(\mathbf{X}: \mathbf{1})$ & $\begin{array}{c}\text { Injection } \\
\text { Volume }(\boldsymbol{\mu L})\end{array}$ & $\begin{array}{c}\text { Inlet Temperature } \\
\left({ }^{\circ} \mathbf{C}\right)\end{array}$ \\
\hline 1 & 230 & 10 & 0.5 & 200 \\
\hline 2 & 280 & 10 & 0.5 & 300 \\
\hline 3 & 230 & 30 & 0.5 & 300 \\
\hline 4 & 280 & 30 & 0.5 & 200 \\
\hline 5 & 230 & 10 & 2 & 300 \\
\hline 6 & 280 & 10 & 2 & 200 \\
\hline 7 & 230 & 30 & 2 & 300 \\
\hline 8 & 280 & 30 & 2 & \\
\hline
\end{tabular}


Supplemental Table 4. Complete list of the 222 opioids and related compounds analyzed using the targeted method along with their retention times and retention indices (Step 4).

\begin{tabular}{|c|c|c|c|c|c|}
\hline Compound & RT (min) & $\mathbf{R I}$ & Compound & RT (min) & RI \\
\hline Tramadol & 2.085 & 2265 & Carfentanil & 10.784 & 3379 \\
\hline Xylazine & 2.342 & 2363 & 3,4-Ethylenedioxy U-47700 & 10.794 & 3380 \\
\hline o-Desmethyl-cis-Tramadol & 2.400 & 2386 & Valeryl Fentanyl & 10.8 & 3385 \\
\hline N-methyl Norfentanyl & 2.470 & 2409 & $\begin{array}{l}\text { 2,2,3,3-tetramethyl- } \\
\text { Cyclopropyl Fentanyl }\end{array}$ & 10.869 & 3385 \\
\hline Norsufentanil & 2.692 & 2471 & p-methyl Cyclopropyl Fentanyl & 11.05 & 3398 \\
\hline Norfentanyl & 2.698 & 2473 & 3,4-Ethylenedioxy U-51754 & 11.167 & 3405 \\
\hline Acetyl norfentanyl & 2.709 & 2483 & N-(Phentermine) Fentanyl & 11.248 & 3408 \\
\hline $\begin{array}{l}\text { N-methyl Cyclopropyl } \\
\text { Norfentanyl }\end{array}$ & 2.714 & 2487 & p-fluoro Crotonyl Fentanyl & 11.295 & 3413 \\
\hline cis-3-methyl Norfentanyl & 2.791 & 2500 & $\alpha^{\prime}$-methoxy Fentanyl & 11.388 & 3419 \\
\hline Butyryl Norfentanyl & 2.902 & 2526 & p-fluoro Valeryl Fentanyl & 11.458 & 3424 \\
\hline N-methyl Norcarfentanil & 3.024 & 2554 & p-Chloroisobutyryl Fentanyl & 11.493 & 3426 \\
\hline Norcarfentanil & 3.589 & 2669 & Octfentanil & 11.609 & 3434 \\
\hline U-48753E & 3.659 & 2682 & $\begin{array}{l}\text { m-fluoro Methoxyacetyl } \\
\text { Fentanyl }\end{array}$ & 11.729 & 3441 \\
\hline p-fluoro 4-ANBP & 4.026 & 2743 & Methoxyacetyl Fentanyl & 11.959 & 3461 \\
\hline $\begin{array}{l}\text { Despropionyl 2'-fluoro o- } \\
\text { Fluorofentanyl }\end{array}$ & 4.207 & 2776 & Cyclobutyl Fentanyl & 12.14 & 3467 \\
\hline 4-ANPP & 4.621 & 2834 & $\begin{array}{c}\text { p-fluoro Methoxyacetyl } \\
\text { Fentanyl } \\
\end{array}$ & 12.274 & 3476 \\
\hline Furanyl norfentanyl & 4.755 & 2847 & Ethoxyacetyl Fentanyl & 12.297 & 3477 \\
\hline $\begin{array}{l}\text { Despropioneyl p- } \\
\text { Fluorofentanyl }\end{array}$ & 5.029 & 2882 & Hexanoyl fentanyl & 12.332 & 3479 \\
\hline $\mathrm{U}-47931 \mathrm{E}$ & 5.075 & 2888 & p-Chlorofentanyl & 12.396 & 3484 \\
\hline U-48520 & 5.134 & 2896 & p-chloro Acrylfentanyl & 12.437 & 3486 \\
\hline $\begin{array}{l}\text { Despropionyl m- } \\
\text { Methylfentanyl }\end{array}$ & 5.268 & 2912 & n-benzyl Furanyl Norfentanyl & 12.862 & 3512 \\
\hline AP-238 & 5.420 & 2929 & o-methoxy Butyryl Fentanyl & 12.95 & 3517 \\
\hline 2,3-seco-Fentanyl & 5.821 & 2973 & Cyclopentyl Fentanyl & 12.956 & 3520 \\
\hline 2-Methyl AP-237 & 5.882 & 2980 & $\begin{array}{l}\text { m-methyl Methoxyacetyl } \\
\text { Fentanyl }\end{array}$ & 12.979 & 3520 \\
\hline MT-45 & 5.915 & 2983 & $\begin{array}{l}\text { o-methyl Methoxyacetyl } \\
\text { Fentanyl }\end{array}$ & 13.026 & 3523 \\
\hline 2-fluoro MT-45 & 6.060 & 2999 & p-Chlorobutyryl Fentanyl & 13.241 & 3535 \\
\hline Fentanyl Methyl Carbamate & 6.480 & 3039 & p-Methoxyfentanyl & 13.463 & 3551 \\
\hline 6-Monoacetylmorphine & 6.559 & 3047 & $\begin{array}{c}\text { p-methyl Methoxyacetyl } \\
\text { Fentanyl }\end{array}$ & 13.51 & 3551 \\
\hline U-47700 & 6.806 & 3071 & p-chloro Cyclopropyl Fentanyl & 13.667 & 3560 \\
\hline Fentanyl Carbamate & 6.812 & 3071 & p-fluoro Cyclopentyl Fentanyl & 13.748 & 3565 \\
\hline Isopropyl U-47700 & 6.818 & 3072 & p-methoxy Acetyl fentanyl & 13.876 & 3573 \\
\hline U-48800 & 7.011 & 3091 & p-methoxy Acrylfentanyl & 13.906 & 3575 \\
\hline Propyl U-47700 & 7.069 & 3096 & Heptanoyl fentanyl & 14.069 & 3584 \\
\hline Benzyl Fentanyl & 7.110 & 3103 & p-Bromofentanyl & 14.256 & 3594 \\
\hline
\end{tabular}




\begin{tabular}{|c|c|c|c|c|c|}
\hline Compound & RT (min) & $\mathbf{R I}$ & Compound & RT (min) & $\mathbf{R I}$ \\
\hline Isobutyryl Fentanyl & 7.215 & 3109 & p-methyl Cyclopentyl fentanyl & 14.268 & 3596 \\
\hline Benzyl Acrylfentanyl & 7.302 & 3116 & p-methoxy Butyryl Fentanyl & 14.39 & 3603 \\
\hline 3,4-Methylenedioxy U-47700 & 7.320 & 3118 & Tetrahydrofuran Fentanyl & 14.424 & 3607 \\
\hline cis-Isofentanyl & 7.523 & 3135 & $\begin{array}{c}\text { p-fluoro Tetrahydrofuran } \\
\text { Fentanyl }\end{array}$ & 14.652 & 3618 \\
\hline Thienyl Fentanyl & 7.576 & 3139 & p-chloro Valeryl Fentanyl & 14.745 & 3624 \\
\hline N-benzyl p-fluoro Norfentanyl & 7.664 & 3144 & m-fluoro Furanyl Fentanyl & 14.821 & 3631 \\
\hline Remifentanil & 7.955 & 3174 & $\begin{array}{c}\text { Furanyl fentanyl 3- } \\
\text { furancarboxamide isomer }\end{array}$ & 14.827 & 3632 \\
\hline Remifentanil Acid & 8.037 & 3178 & Cyclohexyl Fentanyl & 14.914 & 3634 \\
\hline o-Fluoroisobutyryl Fentanyl & 8.153 & 3188 & 4-Phenyl fentanyl & 14.955 & 3636 \\
\hline o-fluoro Acrylfentanyl & 8.171 & 3189 & 4-phenyl U-51754 & 15.089 & 3643 \\
\hline AH 7921 & 8.176 & 3189 & o-fluoro Furanyl Fentanyl & 15.124 & 3645 \\
\hline m-Fluoroisobutyryl Fentanyl & 8.206 & 3192 & Furanyl fentanyl & 15.235 & 3652 \\
\hline U-49900 & 8.241 & 3198 & Cyclopentenyl fentanyl & 15.258 & 3653 \\
\hline Oxycodone & 8.274 & 3198 & $\begin{array}{c}\text { p-fluoro Furanyl Fentanyl 3- } \\
\text { furacncarboxamide }\end{array}$ & 15.353 & 3659 \\
\hline$\beta$-methyl Fentanyl & 8.299 & 3200 & N-Benzyl phenyl norfentanyl & 15.404 & 3661 \\
\hline Sufentanil & 8.433 & 3210 & p-fluoro Furanyl Fentanyl & 15.474 & 3666 \\
\hline$\beta$-methyl Acetyl Fentanyl & 8.497 & 3214 & $\begin{array}{c}\text { p-chloro Methoxyacetyl } \\
\text { Fentanyl } \\
\end{array}$ & 15.631 & 3675 \\
\hline Pivaloyl Fentanyl & 8.579 & 3221 & Alfentanil & 15.672 & 3677 \\
\hline FIBF & 8.580 & 3222 & p-methoxy Valeryl fentanyl & 15.928 & 3692 \\
\hline $\begin{array}{l}\text { N-benzyl p-fluoro Cyclopropyl } \\
\text { norfentanyl }\end{array}$ & 8.596 & 3225 & $\begin{array}{l}\text { p-methyl Tetrahydrofuran } \\
\text { fentanyl }\end{array}$ & 16.03 & 3697 \\
\hline U-51754 & 8.608 & 3230 & o-methyl Furanyl fentanyl & 16.173 & 3706 \\
\hline trans-3-methyl Fentanyl & 8.678 & 3231 & m-methyl Furanyl fentanyl & 16.197 & 3707 \\
\hline o-Fluorofentanyl & 8.712 & 3231 & p-chloro Cyclobutyl fentanyl & 16.319 & 3714 \\
\hline 2'-fluoro-o-fluorofentanyl & 8.724 & 3232 & p-methyl Furanyl fentanyl & 16.954 & 3749 \\
\hline Fentanyl & 8.847 & 3243 & 2',5'-dimethoxy Fentanyl & 17.001 & 3751 \\
\hline m-Fluorofentanyl & 8.870 & 3243 & N-(2C-TFM) Fentanyl & 17.024 & 3752 \\
\hline Acetyl fentanyl & 8.941 & 3249 & N-(2-APB) Fentanyl & 17.03 & 3753 \\
\hline Benzyl Carfentanil & 8.946 & 3249 & N-(MDA) Fentanyl & 17.141 & 3759 \\
\hline Acrylfentanyl & 8.946 & 3250 & N-(2C-D) Fentanyl & 17.223 & 3764 \\
\hline trans-3-methyl Thiofentanyl & 9.020 & 3254 & p-chloro Cyclopentyl fentanyl & 17.397 & 3773 \\
\hline $\begin{array}{l}\text { N,N-Dimethylamido- } \\
\text { despropionyl fentanyl }\end{array}$ & 9.039 & 3255 & N-(6-APB) Fentanyl & 17.438 & 3775 \\
\hline Thiofentanyl & 9.077 & 3259 & N-(2,5-DMA) Fentanyl & 17.444 & 3777 \\
\hline cis-3-methyl Fentanyl & 9.097 & 3266 & Tetrahydrothiophene fentanyl & 17.514 & 3779 \\
\hline p-methyl Isobutyryl Fentanyl & 9.150 & 3267 & N-(DOM) Fentanyl & 17.578 & 3782 \\
\hline$\alpha^{\prime}$-methyl Butyryl Fentanyl & 9.167 & 3267 & $\begin{array}{l}\text { p-methoxy Methoxyacetyl } \\
\text { fentanyl } \\
\end{array}$ & 17.584 & 3784 \\
\hline Heroin & 9.214 & 3269 & $\mathrm{~N}-(2 \mathrm{C}-\mathrm{iP})$ Fentanyl & 17.753 & 3793 \\
\hline p-Fluorofentanyl & 9.226 & 3274 & N-(2C-E) Fentanyl & 17.759 & 3793 \\
\hline
\end{tabular}




\begin{tabular}{|c|c|c|c|c|c|}
\hline Compound & RT (min) & RI & Compound & RT (min) & RI \\
\hline Metodesnitazene & 9.343 & 3279 & $\begin{array}{c}\text { Tetrahydrofuran Fentanyl 3- } \\
\text { THFcarboximamide }\end{array}$ & 17.8 & 3796 \\
\hline p-fluoro Acrylfentanyl & 9.389 & 3282 & o-isopropyl Furanyl fentanyl & 17.974 & 3805 \\
\hline o-Fluorobutyryl Fentanyl & 9.453 & 3287 & Phenyl fentanyl & 18.022 & 3807 \\
\hline Butyryl Fentanyl & 9.474 & 3289 & N-(DOET) Fentanyl & 18.173 & 3815 \\
\hline p-Fluoro acetyl Fentanyl & 9.482 & 3289 & N-(2C-P) Fentanyl & 18.971 & 3858 \\
\hline 4'-Fluorofentanyl & 9.482 & 3289 & p-chloro Furanyl fentanyl & 18.989 & 3859 \\
\hline cis-3-methyl Thiofentanyl & 9.534 & 3293 & N-(6-APDB) Fentanyl & 19.07 & 3864 \\
\hline m-Methylfentanyl & 9.569 & 3298 & o-methoxy Furanyl Fentanyl & 19.14 & 3867 \\
\hline m-Fluorobutyryl Fentanyl & 9.604 & 3298 & Thiophene fentanyl & 19.245 & 3873 \\
\hline o-Methylfentanyl & 9.622 & 3300 & N-(2C-G) Fentanyl & 19.292 & 3875 \\
\hline Isovaleryl Fentanyl & 9.627 & 3300 & o-methyl Phenyl fentanyl & 19.373 & 3880 \\
\hline$\alpha$-methyl Fentanyl & 9.715 & 3306 & Phenylacetyl fentanyl & 19.834 & 3904 \\
\hline Cyclopropyl Fentanyl & 9.767 & 3308 & p-Toluoyl fentanyl & 20.079 & 3917 \\
\hline o-methyl Acrylfentanyl & 9.773 & 3310 & $\begin{array}{l}\text { p-methoxy Tetrahydrofuran } \\
\text { fentanyl }\end{array}$ & 20.364 & 3932 \\
\hline$\alpha$-methyl Acetyl Fentanyl & 9.913 & 3320 & $\beta^{\prime}$-Phenyl fentanyl & 20.86 & 3959 \\
\hline m-methyl Acetyl fentanyl & 9.919 & 3320 & N-(DOBU) Fentanyl & 20.982 & 3965 \\
\hline 3'-methyl Fentanyl & 9.919 & 3320 & Noscapine & 21.224 & 3977 \\
\hline o-methyl Acetyl fentanyl & 9.923 & 3321 & p-methoxy Furanyl fentanyl & 21.431 & 3989 \\
\hline cis-3-methyl Butyryl Fentanyl & 9.930 & 3321 & Flunitazene & 21.799 & 4009 \\
\hline p-Methylfentanyl & 9.971 & 3323 & N-(2C-C) Fentanyl & 22.008 & 4019 \\
\hline Etodesnitazene & 9.980 & 3324 & N-(3,4,5-TMA) Fentanyl & 22.392 & 4040 \\
\hline$\alpha$-methyl Thiofentanyl & 10.012 & 3326 & N-(DOC) Fentanyl & 22.404 & 4040 \\
\hline $\begin{array}{l}\text { 4'-fluoro, p-fluoro-trans-3- } \\
\text { methyl Fentanyl }\end{array}$ & 10.059 & 3329 & N-(2C-B) Fentanyl & 23.931 & 4121 \\
\hline p-Fluorobutyryl Fentanyl & 10.112 & 3333 & Etizolam & 24.097 & 4130 \\
\hline Isodesnitazene & 10.126 & 3334 & Phenoxyacetyl fentanyl & 24.188 & 4135 \\
\hline 4'-methyl Fentanyl & 10.129 & 3334 & N-(DOB) Fentanyl & 24.31 & 4141 \\
\hline 3'-methyl Acetyl fentanyl & 10.141 & 3335 & Brorphine & 24.916 & 4173 \\
\hline Senecioylfentanyl & 10.146 & 3336 & N-(3-ethylindole) Norfentanyl & 24.934 & 4174 \\
\hline Quinine & 10.208 & 3340 & N-(2C-T-4) Fentanyl & 25.4 & 4199 \\
\hline 4'-methyl Acetyl Fentanyl & 10.216 & 3340 & N-(2C-T) Fentanyl & 25.644 & 4212 \\
\hline p-methyl Acetyl Fentanyl & 10.234 & 3342 & N-(2C-T-2) Fentanyl & 25.826 & 4221 \\
\hline p-methyl Acryl Fentanyl & 10.246 & 3342 & N-(2C-I) Fentanyl & 25.867 & 4222 \\
\hline p-fluoro Cyclopropyl Fentanyl & 10.415 & 3354 & N-(DOI) Fentanyl & 26.175 & 4240 \\
\hline$\alpha$-methyl Butyryl Fentanyl & 10.420 & 3354 & Benzodioxole fentanyl & 26.951 & 4281 \\
\hline 2'-methyl Fentanyl & 10.496 & 3360 & Metonitazene & 26.996 & 4283 \\
\hline m-methyl Cyclopropyl fentanyl & 10.560 & 3364 & N-(2C-T-7) Fentanyl & 27.044 & 4285 \\
\hline o-methyl Cyclopropyl Fentanyl & 10.630 & 3368 & Isotonitazene & 28.075 & 4341 \\
\hline p-methyl Butyryl fentanyl & 10.636 & 3369 & N-(2C-B-fly) Fentanyl & 32.752 & 4601 \\
\hline 2'-methyl Acetyl fentanyl & 10.724 & 3375 & N-(3C-B-fly) Fentanyl & 33.205 & 4623 \\
\hline Crotonyl Fentanyl & 10.735 & 3377 & N-(2C-N) Fentanyl & 33.665 & 4654 \\
\hline
\end{tabular}


Supplemental Table 5. Instrumental parameters for the general confirmation method used for comparison purposes (Step 5).

\begin{tabular}{|c|c|}
\hline Column & DB-5 \\
\hline Temperature Program & $\begin{array}{l}\text { 1) } 180{ }^{\circ} \mathrm{C} \text { starting temperature } \\
\text { 2) Ramp } 30^{\circ} \mathrm{C} \min ^{-1} \text { to } 280^{\circ} \mathrm{C} \\
\text { 3) Hold } 8 \mathrm{~min}\end{array}$ \\
\hline Flow Rate & $\begin{array}{l}\text { 1) } 1.8 \mathrm{~mL} \mathrm{~min} \min ^{-1} \text {, hold for } 5 \mathrm{~min} \\
\text { 2) Ramp } 0.5 \mathrm{~mL} \mathrm{~min}^{-1} \text { to } 2.0 \mathrm{~mL} \mathrm{~min}-1 \\
\text { 3) Hold at } 2.0 \mathrm{~mL} \mathrm{~min}^{-1}\end{array}$ \\
\hline Injection Volume & $1.0 \mu \mathrm{L}$ \\
\hline Inlet Temperature & $250^{\circ} \mathrm{C}$ \\
\hline Split Ratio & $50: 1$ \\
\hline Transfer Line & $280^{\circ} \mathrm{C}$ \\
\hline Quad Temperature & $150^{\circ} \mathrm{C}$ \\
\hline Source Temperature & $230^{\circ} \mathrm{C}$ \\
\hline Tune Mode & stune \\
\hline Solvent Delay & $1.0 \mathrm{~min}$ \\
\hline Mass Scan Range & $m / 240-m / 2550$ \\
\hline Threshold & 150 \\
\hline Scan Speed & $\mathrm{N}=2$ \\
\hline Total Run Time & $11.33 \mathrm{~min}$ \\
\hline
\end{tabular}

Supplemental Table 6. Metrics for the comparison of the targeted method to the general screening method (Step 5). Uncertainties represent the standard deviation of triplicate measurements. The notation "NEP" indicates a non-eluting peak. Compounds are listed in the order they elute on the DB-5 column (general confirmatory method). The number in parenthesis to right of the name is the elution order on the DB-200 column.

\begin{tabular}{|c|c|c|c|c|c|c|}
\hline & \multicolumn{2}{|c|}{ Approximate LOD $\left(\mu \mathrm{g} \mathrm{mL}^{-1}\right)$} & \multicolumn{2}{|c|}{$\begin{array}{c}\text { \%RTD Between } \\
\text { Neighboring Compounds }\end{array}$} & \multicolumn{2}{|c|}{ Peak Area (Counts/s) } \\
\hline & Targeted & General & Targeted & General & Targeted & General \\
\hline m-FIBF (1) & 1 & 10 & $\begin{array}{c}5.45 \\
( \pm 0.04)\end{array}$ & $1.29( \pm 0.07)$ & $\begin{array}{c}1.1 \times 10^{6} \\
\left( \pm 3.6 \times 10^{4}\right)\end{array}$ & $\begin{array}{c}2.0 \times 10^{5} \\
\left( \pm 3.3 \times 10^{4}\right)\end{array}$ \\
\hline p-FIBF (2) & 1 & 10 & $\begin{array}{c}2.02 \\
( \pm 0.04)\end{array}$ & $4.60( \pm 0.00)$ & $\begin{array}{c}1.2 \times 10^{6} \\
\left( \pm 3.9 \times 10^{4}\right)\end{array}$ & $\begin{array}{c}2.5 \times 10^{5} \\
\left( \pm 2.1 \times 10^{4}\right)\end{array}$ \\
\hline Fentanyl (3) & 1 & 25 & $\begin{array}{c}11.63 \\
( \pm 0.04)\end{array}$ & $9.89( \pm 0.01)$ & $\begin{array}{c}1.6 \times 10^{6} \\
\left( \pm 5.1 \times 10^{4}\right)\end{array}$ & $\begin{array}{c}3.2 \times 10^{5} \\
\left( \pm 2.3 \times 10^{4}\right)\end{array}$ \\
\hline $\begin{array}{l}\text { Methoxyacetyl } \\
\text { Fentanyl (7) }\end{array}$ & 10 & 25 & $\begin{array}{c}27.73 \\
( \pm 0.02)\end{array}$ & 0 & $\begin{array}{c}1.2 \times 10^{6} \\
\left( \pm 3.3 \times 10^{4}\right)\end{array}$ & $\begin{array}{c}3.4 \times 10^{5} \\
\left( \pm 7.0 \times 10^{3}\right)\end{array}$ \\
\hline $\begin{array}{l}\text { Cyclopropyl } \\
\text { Fentanyl (4) }\end{array}$ & 1 & 25 & $\begin{array}{c}9.84 \\
( \pm 0.03)\end{array}$ & 0 & $\begin{array}{c}1.5 \times 10^{6} \\
\left( \pm 5.3 \times 10^{4}\right)\end{array}$ & $\begin{array}{c}3.1 \times 10^{5}( \pm 4.1 \\
\left.\times 10^{3}\right)\end{array}$ \\
\hline $\begin{array}{c}\text { Crotonyl } \\
\text { Fentanyl (5) }\end{array}$ & 10 & 25 & $\begin{array}{c}10.49 \\
( \pm 0.00)\end{array}$ & $1.32( \pm 0.04)$ & $\begin{array}{c}1.5 \times 10^{6} \\
\left( \pm 6.2 \times 10^{4}\right)\end{array}$ & $\begin{array}{c}3.1 \times 10^{5} \\
\left( \pm 4.1 \times 10^{3}\right)\end{array}$ \\
\hline Carfentanil (6) & 10 & 25 & $\begin{array}{c}0.83 \\
( \pm 0.03)\end{array}$ & $\begin{array}{c}17.55 \\
( \pm 1.68)\end{array}$ & $\begin{array}{c}1.1 \times 10^{6} \\
\left( \pm 3.5 \times 10^{4}\right)\end{array}$ & $\begin{array}{c}2.7 \times 10^{5} \\
\left( \pm 1.0 \times 10^{4}\right)\end{array}$ \\
\hline $\begin{array}{c}\text { Furanyl } \\
\text { Fentanyl (8) }\end{array}$ & 1 & 25 & $\begin{array}{c}40.01 \\
( \pm 0.05)\end{array}$ & $0.76( \pm 0.05)$ & $\begin{array}{c}1.2 \times 10^{6} \\
\left( \pm 3.1 \times 10^{4}\right)\end{array}$ & $\begin{array}{c}2.9 \times 10^{5} \\
\left( \pm 8.3 \times 10^{2}\right)\end{array}$ \\
\hline Etizolam (10) & 25 & 50 & $\begin{array}{c}13.45 \\
( \pm 0.03)\end{array}$ & $\begin{array}{c}40.09 \\
( \pm 8.09)\end{array}$ & $\begin{array}{c}2.0 \times 10^{5} \\
\left( \pm 6.0 \times 10^{4}\right)\end{array}$ & $\begin{array}{c}1.2 \times 10^{5} \\
\left( \pm 1.1 \times 10^{4}\right)\end{array}$ \\
\hline Noscapine (9) & 10 & 50 & $\begin{array}{c}11.91 \\
( \pm 0.04)\end{array}$ & N/A & $\begin{array}{c}1.1 \times 10^{6} \\
\left( \pm 2.6 \times 10^{4}\right) \\
\end{array}$ & $\begin{array}{c}2.5 \times 10^{5} \\
\left( \pm 1.7 \times 10^{4}\right)\end{array}$ \\
\hline $\begin{array}{l}\text { Benzodioxole } \\
\text { Fentanyl (11) }\end{array}$ & 25 & NEP & N/A & N/A & $\begin{array}{c}5.3 \times 10^{5} \\
\left( \pm 2.0 \times 10^{4}\right)\end{array}$ & NEP \\
\hline
\end{tabular}

